\title{
A general protocol to afford enantioenriched linear homoprenylic
}

\section{amines $\dagger$}

\author{
Irene Bosque, Francisco Foubelo and Jose C. Gonzalez-Gomez* \\ Departamento de Química Orgánica, Facultad de Ciencias and Instituto de Síntesis Orgánica (ISO), \\ Universidad de Alicante,Apdo.99,03080 Alicante,Spain.E-mail: josecarlos.gonzalez@ua.es \\ $\dagger$ Electronic Supplementary Information (ESI) available: Copies of ${ }^{1} \mathrm{H}$ and ${ }^{13} \mathrm{C}$ NMR spectra for all \\ reported compounds, HPLC chromatograms and details for the determination of absolute configuration of \\ selected examples. Crystal data file for compound ent-2b (CCDC 953178) is also provided.
}

\begin{abstract}
The reaction of a readily obtained chiral branched homoprenylamonium salt with a range of aldehydes, including aliphatic substrates, affords the corresponding linear isomers in good yields and enantioselectivities.
\end{abstract}

\section{Introduction}

Allylation of imines is one of the most valuable tools to build enantioenriched homoallylic amines, using either catalytic ${ }^{1}$ or non-catalytic ${ }^{2}$ methods. In addition to the challenge posed by the stereoselection, regioselectivity is a very important issue in allylation reactions. Generally, $\gamma$-substituted allylic reagents furnish the corresponding branched products ( $\gamma$-adducts) with high selectivity. In particular, very few examples of $\alpha$-regioselective prenylation of imines have been reported. The first useful protocol was reported by $\mathrm{H}$. Yamamoto in 1996 and consists in the addition of in-situ generated prenyl barium reagent to imines at $0{ }^{\circ} \mathrm{C} .{ }^{3}$ Although this elegant procedure showed excellent levels of regioselectivity in the examples examined, it has some practical limitations related to the formation and manipulation of activated (Rieke) barium under anhydrous conditions. In the past two decades very little progress has been made in this area. Recently it was reported that prenylhafnium reagent, formed from prenylSnBu $3 / \mathrm{HfCl}_{4}$, adds to imines in propionitrile with excellent $\alpha$-selectivity. ${ }^{4}$ More recently, it was found that prenyl zinc reagent in combination with 1,3-dimethyl-2imidazolidinone at $120^{\circ} \mathrm{C}$ can also be used for the $\alpha$-addition to imines. ${ }^{5}$ Furthermore, the cross-coupling of imines with 2-methyl-3-buten-2-ol, mediated by an in-situ formed low-valent titanium reagent, has also been successfully used. ${ }^{6}$ Very recently, the linear prenylation of imines has also been accomplished under transfer hydrogenation conditions. $^{7}$ Surprisingly, to our best knowledge, only one example of $\alpha$-prenylation of 
imines with moderate enantioselectivity $\left(80: 20\right.$ er) has been reported to date. ${ }^{3}$ Enantioenriched homoprenyl amines have been recently prepared by $\alpha$-deprotonation of amines with $\mathrm{n}-\mathrm{BuLi} /(-)$-sparteine, to give a chiral organolithium that was quenched with prenyl bromide. This method has been successfully applied to N-Boc pyrrolidine and benzylamines. ${ }^{8}$

Homoprenylsulfonamides have been extensively used for the synthesis of pyrrolidines through 5 -endo-trig cyclizations promoted by triflic acid ${ }^{9}$ or iodine. ${ }^{10}$ Furthermore, the utility of homoprenyl amine derivatives as building blocks of bioactive natural compounds has also been illustrated under cationic ${ }^{11}$ or radical ${ }^{12}$ conditions. Given that the protocols available for $\alpha$-regioselective prenylation of imines remain scarce, the synthetic applications of the corresponding linear amines are still underexploited. In this context we decided to develop an alternative method that allows access to a variety of enantioenriched linear homoprenylamines from an easily available chiral substrate.

Our approach is based on the precedented 2-azonia-Cope rearrangement of chiral iminiums derived from branched homoallylic primary amines. This strategy was first employed by using a derivative of $(1 R)$-camphorquinone as a chiral template ${ }^{13}$ and more recently in catalytic methods that use chiral Brønsted acids as ligands for the asymmetric induction via a chiral ion-pair. ${ }^{14}$ To our surprise, this approach has never been used as a general method to generate homoprenylic amines. ${ }^{15} \mathrm{We}$ reasoned that chiral $\gamma$-adducts, easily available from the addition of prenyl organometallic reagents to imine derivatives, could act as convenient aminoprenyl donors to other aldehydes. In our approach, the geminal methyl groups are expected to sterically promote the rearrangement to an iminium ion with an internal more stable double bond (Scheme 1). In this model it is assumed that the $(E)$-iminium intermediate rearranges through a closed chair-like transition state in which the bulkier substituent $\left(\mathrm{R}^{1}\right)$ is placed in equatorial position. The fact that this favored transition state involves two gauche interactions of the $\mathrm{R}^{1}$ group with the geminal methyl groups anticipated a more challenger enantioselection than in other related transfer aminoallylations. 


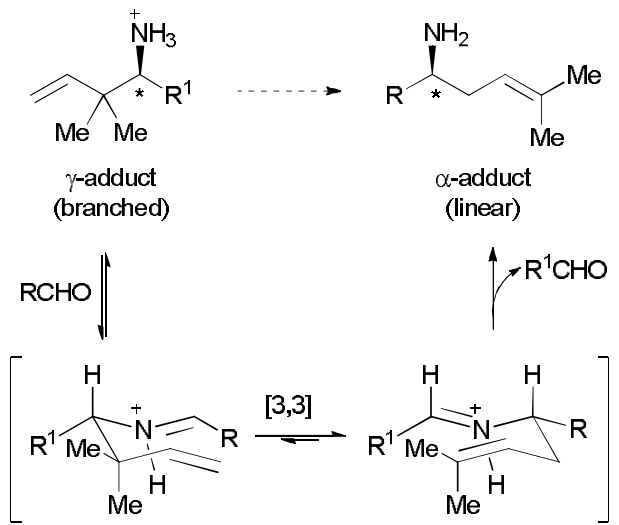

Scheme 1. Working model for enantioselective transfer aminoprenylation of aldehydes.

\section{Results and discussion}

To prepare the chiral donors we used the indium mediated one-pot protocol recently developed in our research group for the diastereoselective aminoallylation of aldehydes. ${ }^{16}$ The corresponding branched homoprenylic sulfinamines were obtained with high $\gamma$-regio and stereoselectivity. After conventional acidic deprotection, the corresponding enantioenriched ${ }^{17}$ amine hydrochlorides $\mathbf{3 a}$ and $\mathbf{3 b}$ were obtained in quantitative yield. (Scheme 2).

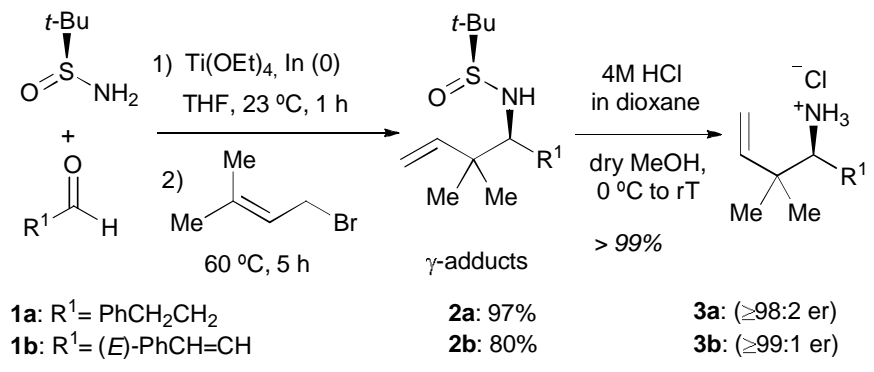

Scheme 2. Enantioenriched homoprenyl amine donors.

Initially, we screened both aminoprenyl chiral donors using 3-phenylpropanal as acceptor. The conditions chosen were very simple. The corresponding $\gamma$-adduct $\mathbf{2}$ was in-situ deprotected and after removal of all volatile compounds under vacuum, a solution of 3-phenylpropanal (1.2 equiv) in the appropriate solvent was added to the chiral donor 3 and submitted to the reaction conditions indicated in Table 1. While the best yield in the sigmatropic rearrangement at $25^{\circ} \mathrm{C}$ was obtained in $\mathrm{CH}_{2} \mathrm{Cl}_{2}$ with the donor 3a (entry 1), the best enantioselectivity was obtained using the donor derived from cinnamaldehyde ( $\mathbf{3 b}$, entry 2 ). In the latter case, it was necessary to quench the reaction mixture with $\mathrm{HONH}_{2} \cdot \mathrm{AcOH}$ in $\mathrm{MeOH},{ }^{13}$ probably due to the better stability of the conjugate iminium formed after the rearrangement. We were encouraged by the 
result obtained with $\mathbf{3 b}$ because this donor was obtained enantiomerically pure in excellent yield, and the absolute configuration of its enantiomer (ent-2b) was ascertained by X-ray crystal diffraction analysis (see ESI). More importantly, this result proved that compound $\mathbf{3 b}$ could act as chiral aminoprenyl transfer agent to different aldehydes. In order to avoid the quenching with $\mathrm{HONH}_{2} \cdot \mathrm{AcOH}$, it was examined the addition of 5 equivalents of $\mathrm{H}_{2} \mathrm{O}$ to the reaction mixture but only starting $\mathbf{3 b}$ was recovered (entry 3 ). The reaction was found to proceed with similar enantioselectivity in toluene but in lower yield (entry 4). When the reaction was run at $40{ }^{\circ} \mathrm{C}$, the same enantioselectivity was obtained for $\mathbf{4 a}$ but in a shorter reaction time (12 h, entry 5). We examined the addition of different molecular sieves to the reaction mixture (entries 6-8), however we observed slightly lower yields and enantioselectivities in all cases. More importantly, we also demonstrate that the reaction proceed in good yield in the presence of substoichiometric amounts of camphorsulfonic acid (entries 9, 10). Unfortunately, the enantioselectivities obtained with both enantiomeric chiral Brønsted acids were lower than with the hydrochloride $\mathbf{3 b}$.

Table 1. Screening of different reaction conditions.

$\begin{array}{cclcccc} & \end{array}$

Having found a simple and reliable set of conditions for the enantioselective aminoprenylation of aldehydes we set about determining the scope and limitations. A range of aldehydes was examined, affording the results shown in Table 2. Given that enolizable aliphatic aldehydes would furnish iminium ions which are in equilibrium with their enamines, these substrates are more challengers. We were pleased to find that 
this subclass of aldehydes (Table 2, entries 1-5) were suitable substrates for this protocol, affording the corresponding linear homoprenylic amines in good yields and moderate to good enantioselectivities. Interestingly the use of the chiral $(S)$-citronellal resulted in a match (entry 5) and mismatched (entry 4) combination with the chiral donor (ent-3b or $\mathbf{3 b}$, respectively) that impacted both, the isolated yield and the enantioselectivity. A limitation to this protocol was encountered with benzaldehyde, which gave very little conversion to isolate a pure product. However, cinnamaldehyde (entry 6) and aromatic aldehydes with diverse electron-withdrawing substituents (entries 7-12) furnished the corresponding enantioenriched products ( $\geq 89: 11$ er) in decent yields. Importantly, our protocol tolerates various functional groups -including nitro, halogens, alkenes and alkoxycarbonyl groups- in the reactive aldehyde. We thus decided to take advantage of this chemoselectivity. When 3-methoxycarbonyl-propanal was used in our standard protocol, the corresponding $\gamma$-lactam was directly obtained (entry 13). However, the use of 5-ethoxycarbonyl pentanal lead to the formation of the corresponding acyclic aminoprenyl derivative in excellent yield (entry 14). More interestingly, when 6-oxoheptanal was used, the aminoprenylation was followed by intramolecular iminium formation. Upon reduction with $\mathrm{NaCNBH}_{3}$, the corresponding cis-2,7-azepane derivative 4o was obtained in reasonable overall yield and good enantioselectivity (entry 15).

Table 2. Substrate scope.

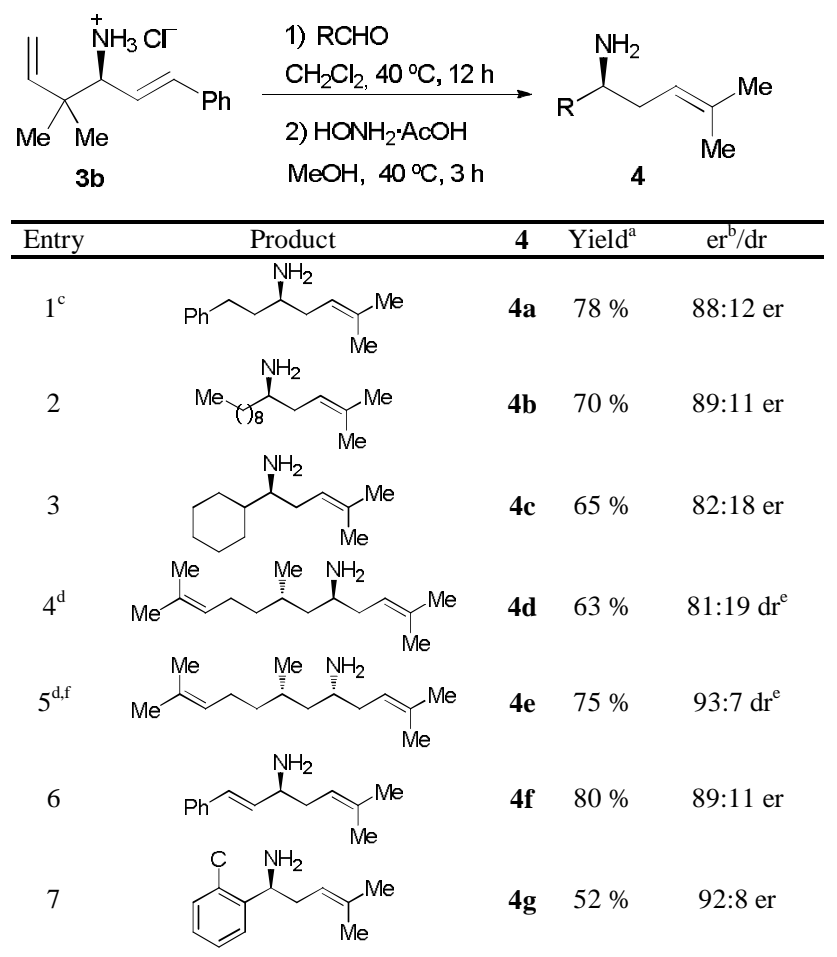


8

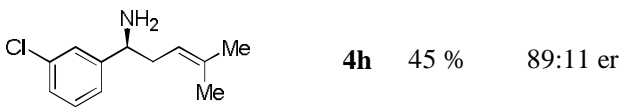

$9^{c}$

10

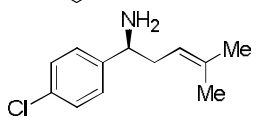

4i $30 \% \quad 89: 11$ er

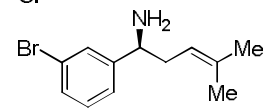

4j $50 \% \quad 89: 11$ er

11

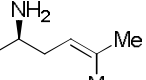

4k $83 \% \quad 90: 10$ er

12

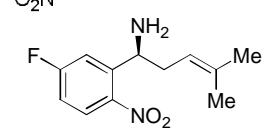

$41 \quad 60 \% \quad 94: 6$ er

$13^{\mathrm{c}}$<smiles>CC=CCC1CCC(=O)N1</smiles>

4m $75 \%$

$88: 12$ er

$14^{\mathrm{c}}$<smiles>CCOC(=O)[AlH]C(N)CC=C(C)C</smiles>

$15^{\mathrm{c}, \mathrm{g}}$

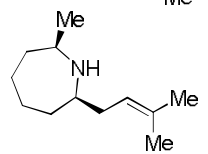

$40 \quad 37 \% \quad 86: 14$ er

[a] Isolated yields after column chromatography. [b] Determined by HPLC analysis (CHIRALCEL OD-H). [c] The enantiomer was also obtained from ent-3b with the same selectivity. [d] $(S)$-citronellal with 98:2 er was used. [e] Determined by ${ }^{13} \mathrm{C}-\mathrm{NMR}$ analysis. [f] ent-3b was used as chiral donor. [g] $\mathrm{NaCNBH}_{3}$ was used to quench the reaction.

The assignment of the absolute configuration of the major enantiomer of amine 4a was performed by ${ }^{1} \mathrm{H}-\mathrm{NMR}$ analysis of its $(R)$-MPA amide, with and without $\mathrm{Ba}\left(\mathrm{ClO}_{4}\right)_{2}$. As described by the group of Riguera, the addition of $\mathrm{Ba}^{2+}$ to the NMR sample of $4 \mathbf{a}$ should facilitate the formation of a chelate. ${ }^{18}$ This equilibrium shift from an antiperiplanar to a synperiplanar conformation leads to an increase shielding of the vinylic and methyl signals by the phenyl group of the MPA auxiliary (Figure 1). The same analysis was performed to revealed the absolute configuration of the major enantiomers of $\mathbf{4 b}$ and $\mathbf{4 k}$ (see ESI for details). Importantly, the inversion of the configuration at the stereogenic center from donor $(S)$-3b to the major enantiomer of $\mathbf{4}$ is predicted by our working model (Scheme 1). 


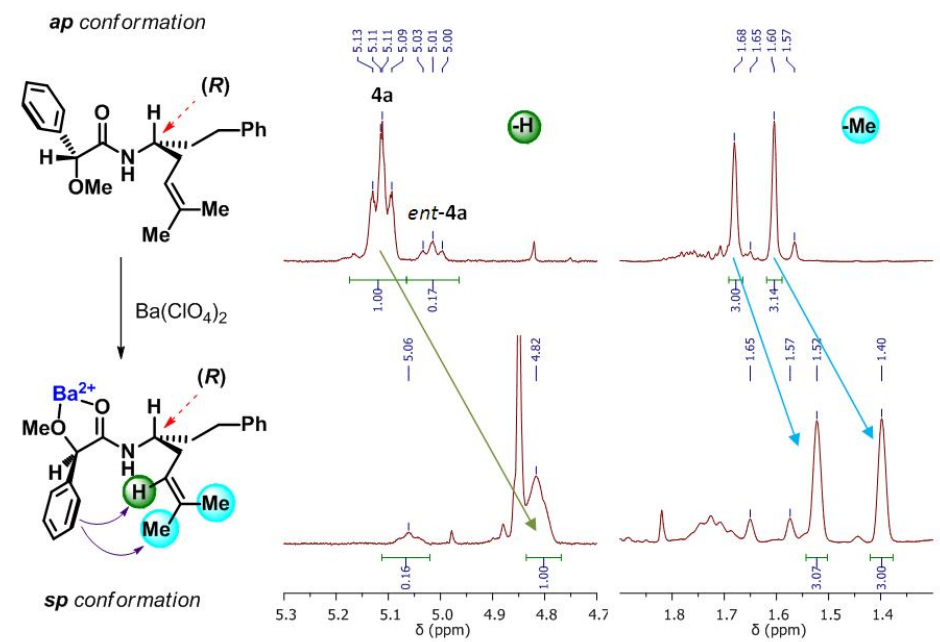

Figure 1 Absolute configuration of $\mathbf{4 a}$ by ${ }^{1} \mathrm{H}-\mathrm{NMR}$ analysis of its $(R)$-MPA amide.

It is worth noting that although this protocol requires the use of stoichiometric amounts of chiral tert-butyl sulfinamide, some practical procedures have been described for recycling this auxiliary upon removal. ${ }^{19}$ Moreover, the ready availability of both antipodes of tert-butyl sulfinamide allows access to both enantiomeric forms of the corresponding linear homoprenylamine. ${ }^{20}$

The potential of the enantioenriched homoprenylamines as building blocks of pyrrolidines was illustrated by preparing the p-nosylate of $\mathbf{4 a}$ and $\mathbf{4 n}$, which upon treatment with substoichiometric amounts of triflic acid at $0{ }^{\circ} \mathrm{C}$ underwent very smooth and efficient intramolecular hydroamination (Scheme 3). ${ }^{9 \mathrm{a}, 9 \mathrm{~b}}$

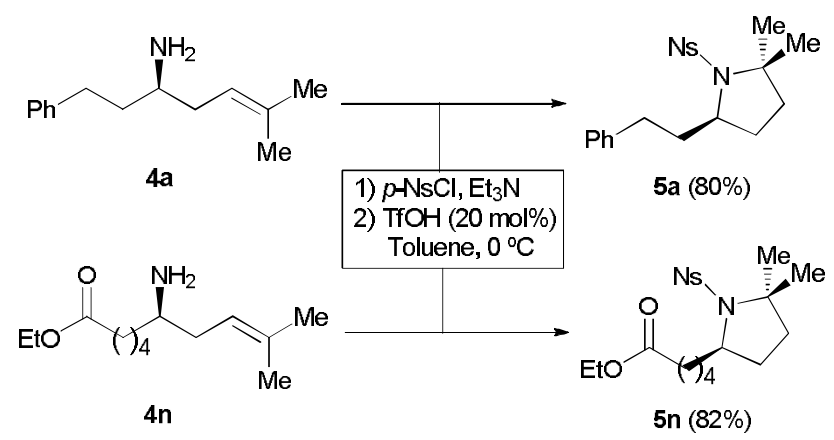

Scheme 3. Pyrrolidine synthesis from compounds $\mathbf{4 a}$ and $\mathbf{4 n}$.

\section{Conclusions}

A general protocol to enantioenriched linear homoprenylic amines has been developed. The procedure is based in the transfer aminoprenylation of aldehydes from a unique chiral branched donor through a 2-azonia-Cope rearrangement. Importantly, 
both enantiomeric forms of the chiral donor are obtained from commercially available starting materials after only two synthetic operations. To the best of our knowledge, this represents the most general and easy to reproduce protocol that gives access to these highly versatile building blocks. Therefore, we hope that this methodology may find wide applications in synthetic organic chemistry.

\section{Experimental}

\section{General Remarks:}

$\left(R_{\mathrm{S}}\right)$-N-tert-Butylsulfinyl amine and its enantiomer were a gift of Medalchemy (> 99\% ee by chiral HPLC on a Chiracel AS column, n-Hexane/i-PrOH 90:10, $1 \mathrm{~mL} / \mathrm{min}$, $\lambda=222 \mathrm{~nm}$ ). TLCs were performed on silica gel $60 \mathrm{~F}_{254}$, using aluminum plates and visualized with phosphomolybdic acid (PMA) or ninhydrine stain. Flash chromatographies were carried out on handpacked columns of silica gel 60 (230- 400 mesh). Melting points are uncorrected. Optical rotations were measured using a polarimeter with a thermally jacketted $5 \mathrm{~cm}$ cell at approximately $20^{\circ} \mathrm{C}$ and concentrations (c) are given in g/100 $\mathrm{mL}$. Infrared analyses were performed with a spectrophotometer equipped with an ATR component; wavenumbers are given in $\mathrm{cm}^{-1}$. GC analyses were obtained with an HP-5 column $(30 \mathrm{~m} \times 0.25 \mathrm{~mm}$, i.d. $\times 0.25 \mu \mathrm{m})$ and an EI (70 EV) detector; the temperature program was as follows: hold at $60{ }^{\circ} \mathrm{C}$ for 3 min, ramp from 60 to $270{ }^{\circ} \mathrm{C}$ at $15^{\circ} \mathrm{C} / \mathrm{min}$, hold at $270{ }^{\circ} \mathrm{C}$ for $10 \mathrm{~min}$. Mass spectra (EI) were obtained at $70 \mathrm{eV}$; and fragment ions in $\mathrm{m} / \mathrm{z}$ with relative intensities (\%) in parentheses. HRMS analyses were also carried out in the electron impact mode (EI) at $70 \mathrm{eV}$ using a quadrupole mass analyzer or in the electrospray ionization mode $\left(\mathrm{ES}^{+}\right)$ using a TOF analyzer. ${ }^{1} \mathrm{H}$ NMR spectra were recorded at 300 or $400 \mathrm{MHz}$, using $\mathrm{CDCl}_{3}$ or $\mathrm{CD}_{3} \mathrm{CN}$ as the solvent and TMS as internal Standard $(0.00 \mathrm{ppm})$; the data is being reported as $(\mathrm{s}=$ singlet, $\mathrm{d}=$ doublet, $\mathrm{t}=$ triplet, $\mathrm{m}=$ multiplet or unresolved, $\mathrm{br}=$ broad signal, coupling constant(s) in $\mathrm{Hz}$, integration). ${ }^{13} \mathrm{C}$ NMR spectra were recorded with ${ }^{1} \mathrm{H}$-decoupling at $101 \mathrm{MHz}$ using the solvent signal as reference (77.16 ppm for $\mathrm{CDCl}_{3}$ ). DEPT-135 experiments were performed to assign $\mathrm{CH}, \mathrm{CH}_{2}$ and $\mathrm{CH}_{3}$.

\section{General procedure for the synthesis of the simple chiral donors $2 \mathrm{a}^{21}{ }^{2 \mathrm{~b}}$ and ent-2b}


To a dry flask were added $\left(R_{\mathrm{S}}\right)$ - $N$-tert-butylsulfinyl amine $(605 \mathrm{mg}, 5.00 \mathrm{mmol})$ followed by indium powder $(713 \mathrm{mg}, 6.25 \mathrm{mmol})$, and the mixture was evacuated and left under Ar atmosphere. Then a solution of the corresponding cynnamaldehyde (726 $\mathrm{mg}, 5.5 \mathrm{mmol})$ in dry THF $(10 \mathrm{~mL})$ was added, followed by Ti(OEt $)_{4}(2.3 \mathrm{~mL}, 10.00$ mmol) and the reaction mixture was stirred under Ar for $1 \mathrm{~h}$ at $23^{\circ} \mathrm{C}$. At this time, 3,3dimethylallyl bromide $(873 \mu \mathrm{L}, 7.5 \mathrm{mmol})$ was added and the reaction mixture was heated to $60^{\circ} \mathrm{C}$ for $3 \mathrm{~h}$. The mixture was allowed to reach room temperature and was carefully added over a stirring mixture of 4:1 EtOAc/brine $(50 \mathrm{~mL})$. The resulted white suspension was filtered through a short pad of Celite, washed with EtOAc and organics were concentrated in vacuo. The resulted suspension was diluted in 4:1 EtOAc/Hexane $(50 \mathrm{~mL})$ and filtered again through Celite. Organics were concentrated and the residue (92:8 dr according ${ }^{1} \mathrm{H}$ NMR) was purified by column chromatography (8:2 Hexane/EtOAc).

\section{$\left(S_{\mathrm{S}}, 1 E, 3 S\right)$-N-tert-Butylsulfinyl-4,4-dimethyl-1-phenylhexa-1,5-dien-3-amine (2b)}

Compound $\mathbf{2 b}$ was obtained according to the general procedure as a white solid (1.218 g, 80\%, 99:1 dr according ${ }^{1} \mathrm{H}$ NMR): mp 94.0 - $95.1{ }^{\circ} \mathrm{C}$. Diasteromeric excess was determined by HPLC of the corresponding benzamide (99:1 er by chiral HPLC on a Chiracel ODH column, n-Hexane/i-PrOH 95:5, $1 \mathrm{~mL} / \mathrm{min}, \lambda=220 \mathrm{~nm}$ ): major enantiomer $t_{\mathrm{R}}=16.3 \mathrm{~min}$, minor enantiomer $t_{\mathrm{R}}=21.1 \mathrm{~min} .[\alpha]^{20}{ }_{\mathrm{D}}+163.4(c) 1.10$, $\left.\mathrm{CHCl}_{3}\right) . \mathrm{R}_{\mathrm{f}}=0.21$ (7:3 Hexane/EtOAc). IR: $v_{\max } / \mathrm{cm}^{-1}$ 3289, 3057, 2958, 2924, 1637, 1364, 1337, 1184, 1059, 996, 984, 912. ${ }^{1} \mathrm{H}$ NMR (300 MHz, $\left.\mathrm{CDCl}_{3}\right) \delta 7.43$ - 7.27 (m, $5 \mathrm{H}), 6.63(\mathrm{~d}, J=15.9 \mathrm{~Hz}, 1 \mathrm{H}), 5.96(\mathrm{dd}, J=15.9,8.8 \mathrm{~Hz}, 1 \mathrm{H}), 5.85(\mathrm{dd}, J=17.4,10.8$ $\mathrm{Hz}, 1 \mathrm{H}), 5.19(\mathrm{dd}, J=10.7,1.0 \mathrm{~Hz}, 1 \mathrm{H}), 5.13(\mathrm{dd}, J=17.5,1.1 \mathrm{~Hz}, 1 \mathrm{H}), 3.71$ (d, $J=8.9$ $\mathrm{Hz}, 1 \mathrm{H}), 3.59$ (br s, 1H), 1.19 (s, 9H), 1.07 (s, 3H), 1.06 (s, 3H). ${ }^{13} \mathrm{C}$ NMR (101 MHz, $\left.\mathrm{CDCl}_{3}\right) \delta 145.9(\mathrm{CH}), 136.8(\mathrm{C}), 135.3(\mathrm{CH}), 128.7(\mathrm{CH}), 127.9(\mathrm{CH}), 126.7(\mathrm{CH})$, $126.4(\mathrm{CH}), 114.6\left(\mathrm{CH}_{2}\right), 63.3(\mathrm{CH}), 55.7(\mathrm{C}), 41.7(\mathrm{C}), 26.3\left(\mathrm{CH}_{3}\right), 22.9\left(\mathrm{CH}_{3}\right), 19.7$ $\left(\mathrm{CH}_{3}\right) . \mathrm{GC} \mathrm{t}_{\mathrm{R}}=16.28 \mathrm{~min}$. LRMS (EI) $\mathrm{m} / \mathrm{z}(\%) 249\left(\mathrm{M}^{+}, 2\right), 230(10), 180(10), 179$ (95), 131 (14), 130 (100), 117 (13), 116 (98), 115 (41), 103 (12), 91 (12), 77 (17), 55 (10). HRMS (EI) calcd for $\mathrm{C}_{18} \mathrm{H}_{27} \mathrm{NOS}-\mathrm{C}_{4} \mathrm{H}_{8} 249.1109$, found 249.1227.

The minor compound $\left(\left(S_{\mathrm{S}}, 1 E, 3 R\right)-\mathbf{2 b}\right)$ was also isolated from the reaction described above as a white solid (106 mg, 7\%): $\mathrm{mp} 45.1-46.8{ }^{\circ} \mathrm{C} .[\alpha]^{20}{ }_{\mathrm{D}}+4.0\left(c 1.06, \mathrm{CHCl}_{3}\right) . \mathrm{R}_{\mathrm{f}}$ $=0.34$ (7:3 Hexane/EtOAc). IR $v_{\max } / \mathrm{cm}^{-1} 3288,3057,2957,2925,1637,1366,1337$, 
1183, 1059, 996, 985, 912. ${ }^{1} \mathrm{H}$ NMR (400 MHz, $\left.\mathrm{CDCl}_{3}\right) \delta 7.44-7.27$ (m, 4H), 7.25 $7.16(\mathrm{~m}, 1 \mathrm{H}), 6.62(\mathrm{~d}, J=15.8 \mathrm{~Hz}, 1 \mathrm{H}), 6.20(\mathrm{dd}, J=15.8,8.3 \mathrm{~Hz}, 1 \mathrm{H}), 5.86(\mathrm{dd}, J=$ $17.4,10.8 \mathrm{~Hz}, 1 \mathrm{H}), 5.14(\mathrm{dd}, J=10.8,1.3 \mathrm{~Hz}, 1 \mathrm{H}), 5.08(\mathrm{dd}, J=17.4,1.3 \mathrm{~Hz}, 1 \mathrm{H}), 3.61$ $(\mathrm{t}, J=8.6 \mathrm{~Hz}, 1 \mathrm{H}), 3.30(\mathrm{~d}, J=9.0 \mathrm{~Hz}, 1 \mathrm{H}), 1.22(\mathrm{~s}, 9 \mathrm{H}), 1.11(\mathrm{~s}, 3 \mathrm{H}), 1.07(\mathrm{~s}, 3 \mathrm{H}) .{ }^{13} \mathrm{C}$ NMR (101 MHz, $\left.\mathrm{CDCl}_{3}\right) \delta 144.4(\mathrm{CH}), 136.9(\mathrm{C}), 133.3(\mathrm{CH}), 128.8(\mathrm{CH}), 128.6$ $(\mathrm{CH}), 127.8(\mathrm{CH}), 126.8(\mathrm{CH}), 114.2\left(\mathrm{CH}_{2}\right), 67.9(\mathrm{CH}), 56.8(\mathrm{C}), 41.9(\mathrm{C}), 24.6\left(\mathrm{CH}_{3}\right)$, $24.5\left(\mathrm{CH}_{3}\right), 22.9\left(\mathrm{CH}_{3}\right) . \mathrm{GC} \mathrm{t}_{\mathrm{R}}=16.89 \mathrm{~min}$. LRMS (EI) $\mathrm{m} / z(\%) 249\left(\mathrm{M}^{+}, 2\right), 230(10)$, 180 (10), 179 (95), 131 (14), 130 (100), 117 (13), 116 (98), 115 (35), 103 (12), 91 (13), 77 (17), 55(10). HRMS (EI) calcd for $\mathrm{C}_{18} \mathrm{H}_{27} \mathrm{NOS}-\mathrm{C}_{4} \mathrm{H}_{8} 249.1109$, found 249.1112.

$\left(R_{\mathrm{S}}, 1 E, 3 R\right)$-N-tert-Butylsulfinyl-4,4-dimethyl-1-phenylhexa-1,5-dien-3-amine (ent2b)

Compound ent-2b was obtained according to the general procedure in similar yield with identical spectroscopic and physical data, except for $[\alpha]^{20}{ }_{\mathrm{D}}-162.6\left(c\right.$ 1.01, $\left.\mathrm{CHCl}_{3}\right)$. In this case $\left(S_{\mathrm{S}}\right)$-N-tert-Butylsulfinyl amine was used.

The minor compound $\left(\left(R_{\mathrm{S}}, 1 E, 3 S\right)-\mathbf{2 b}\right)$ was also isolated from the reaction described above as a white foam. The spectroscopic and physical data was identical to $\left(\left(S_{\mathrm{S}}, 1 E, 3 R\right)-\mathbf{2 b}\right)$, except for $[\alpha]^{20}{ }_{\mathrm{D}}-4.5\left(c 0.96, \mathrm{CHCl}_{3}\right)$.

General procedure for the synthesis of the rearranged amines $4 a-4 l, 4 n$ and lactam 4m

To a solution of $\mathbf{2 b}\left[\left(S_{\mathrm{S}}, 1 E, 3 S\right)-\mathbf{2 b}\right](92 \mathrm{mg}, 0.3 \mathrm{mmol})$ in dry $\mathrm{MeOH}(3.7 \mathrm{~mL})$ at $0{ }^{\circ} \mathrm{C}$ was carefully added a solution of $\mathrm{HCl}$ in 1,4-dioxane (4M, $1.2 \mathrm{mmol}, 300 \mu \mathrm{L})$. The reaction mixture was stirred for $1 \mathrm{~h}$ while reaching the room temperature. After removal of the solvent and volatiles under reduced pressure $\left(t-\mathrm{BuSO}_{2} \mathrm{Me}\right)$, the corresponding hydrochloride $\mathbf{3 b}$ was obtained as a white solid. To a solution of crude hydrochloride $\mathbf{3 b}$ in dry $\mathrm{CH}_{2} \mathrm{Cl}_{2}(2 \mathrm{~mL})$ was added the corresponding aldehyde $(0.36 \mathrm{mmol})$, and the reaction was stirred at $40{ }^{\circ} \mathrm{C}$ for $12 \mathrm{~h}$. The reaction was quenched with a solution of $\mathrm{HONH}_{2} \cdot \mathrm{AcOH}$ in $\mathrm{MeOH}(0.5 \mathrm{M}, 1.4 \mathrm{~mL}) .{ }^{13}$ The reaction mixture was diluted with EtOAc $(5 \mathrm{~mL})$ and $\mathrm{NaOH}(2 \mathrm{M}, 5 \mathrm{~mL})$, and after phase separation, the aqueous phase was extracted with EtOAc ( 3 x $5 \mathrm{~mL})$. Organics were washed with $\mathrm{NaOH}(2 \mathrm{M}, 5 \mathrm{~mL})$ followed by brine $(5 \mathrm{~mL})$, dried over $\mathrm{MgSO}_{4}$, filtered and concentrated under reduced pressure. The residue was purified by column chromatography (gradient from 97:3 
$\mathrm{CH}_{2} \mathrm{Cl}_{2} / \mathrm{MeOH}$ to $\left.90: 10: 0.05 \mathrm{CH}_{2} \mathrm{Cl}_{2} / \mathrm{MeOH} / 20 \% \mathrm{NH}_{4} \mathrm{OH}\right)$ to provide the desired amines $\mathbf{4 a}-\mathbf{4 l}$ and $\mathbf{4 n}$. For the lactam $\mathbf{4 m}$, column chromatography was carried out with 1:3 Hexane/EtOAc. The enantiomers of some arranged products (ent-4a, ent-4m, and ent-4n) were prepared following the same procedure but starting from donor ent-2b.

\section{(R)-6-Methyl-1-phenyl-5-hepten-3-amine (4a)}

Following the general procedure from 3-Phenylpropanal, compound 4a was obtained as a yellow oil after column chromatography (48 mg, $0.234 \mathrm{mmol}, 78 \%$ ): Enantiomeric excess was determined by HPLC of the corresponding benzamide (88:12 er on a Chiracel ODH column, n-Hexane/i-PrOH 95:5, $1 \mathrm{~mL} / \mathrm{min}, \lambda=220 \mathrm{~nm}$ ): major enantiomer $t_{\mathrm{R}}=30.4 \mathrm{~min}$, minor enantiomer $t_{\mathrm{R}}=23.9 \mathrm{~min}$. $[\alpha]^{20}{ }_{\mathrm{D}}-6.2\left(c 0.70, \mathrm{CHCl}_{3}\right)$. $\mathrm{R}_{\mathrm{f}}=0.28\left(9: 1 \mathrm{CH}_{2} \mathrm{Cl}_{2} / \mathrm{MeOH}\right) . \mathrm{IR} v_{\max } / \mathrm{cm}^{-1} 3360,3292,3026,2963,2915,2855,1496$, 1453, 1376, 745, 698. ${ }^{1} \mathrm{H}$ NMR (400 MHz, $\left.\mathrm{CDCl}_{3}\right) \delta 7.32-7.27$ (m, 2H), $7.23-7.14$ (m, 3H), $5.13(\mathrm{ddd}, J=8.2,2.7,1.3 \mathrm{~Hz}, 1 \mathrm{H}), 2.84(\mathrm{ddd}, J=12.8,7.5,5.3 \mathrm{~Hz}, 1 \mathrm{H}), 2.76$ (ddd, $J=13.8,10.3,5.8 \mathrm{~Hz}, 2 \mathrm{H}), 2.65$ (ddd, $J=13.7,10.1,6.2 \mathrm{~Hz}, 2 \mathrm{H}), 2.19$ (dt, $J=$ 12.3, $6.1 \mathrm{~Hz}, 1 \mathrm{H}), 2.15-2.05(\mathrm{~m}, 1 \mathrm{H}), 1.85-1.75(\mathrm{~m}, 1 \mathrm{H}), 1.72(\mathrm{~s}, 3 \mathrm{H}), 1.70-1.65$ $(\mathrm{m}, 1 \mathrm{H}), 1.63(\mathrm{~s}, 3 \mathrm{H}) .{ }^{13} \mathrm{C} \mathrm{NMR}\left(101 \mathrm{MHz}, \mathrm{CDCl}_{3}\right) \delta 142.2(\mathrm{C}), 134.7(\mathrm{C}), 128.5(\mathrm{CH})$, $128.5(\mathrm{CH}), 125.9(\mathrm{CH}), 120.7(\mathrm{CH}), 51.6(\mathrm{CH}), 38.6\left(\mathrm{CH}_{2}\right), 35.9\left(\mathrm{CH}_{2}\right), 32.7\left(\mathrm{CH}_{2}\right)$, $26.0\left(\mathrm{CH}_{3}\right), 18.2\left(\mathrm{CH}_{3}\right) . \mathrm{GC} \mathrm{t}_{\mathrm{R}}=12.36 \mathrm{~min}$. LRMS (EI) $\mathrm{m} / z(\%) 203\left(\mathrm{M}^{+}, 0.1\right), 202$ (0.3), 135 (10), 134 (100), 117 (33), 91 (89), 65 (10). HRMS (EI) calcd for $\mathrm{C}_{14} \mathrm{H}_{21} \mathrm{~N}$ 203.1674, found 203.1726.

\section{(S)-6-Methyl-1-phenyl-5-hepten-3-amine (ent-4a)}

Following the general procedure from 3-Phenylpropanal, compound ent-4a was obtained with identical spectroscopic and physical data as compound 4a except for $[\alpha]_{D}^{20}+6.5\left(c 0.75, \mathrm{CHCl}_{3}\right)$. In this case ent-2b was used as chiral donor.

\section{(R)-2-Methyl-2-tridecen-5-amine (4b)}

Following the general procedure from $n$-decanal, compound $\mathbf{4 b}$ was obtained as a yellow oil after column chromatography (47 mg, $0.210 \mathrm{mmol}, 70 \%)$ : Enantiomeric excess was determined by HPLC of the corresponding (R)-MPA derivative (89:11 er, see ESI). $[\alpha]^{20}{ }_{\mathrm{D}}-5.3\left(c 0.95, \mathrm{CHCl}_{3}\right) . \mathrm{R}_{\mathrm{f}}=0.37\left(9: 1 \mathrm{CH}_{2} \mathrm{Cl}_{2} / \mathrm{MeOH}\right) . \mathrm{IR} v \max / \mathrm{cm}-1$ 3360, 3292, 2957, 2922, 2852, 1619, 1572, 815, $721 \mathrm{~cm}^{-1} .{ }^{1} \mathrm{H}$ NMR (400 MHz, $\mathrm{CDCl}_{3}$ ) 
$\delta 5.15$ (ddd, $J=8.1,2.6,1.3 \mathrm{~Hz}, 1 \mathrm{H}), 2.75$ (br s, $1 \mathrm{H}), 2.20-1.87(\mathrm{~m}, 4 \mathrm{H}), 1.73(\mathrm{~s}, 3 \mathrm{H})$, $1.63(\mathrm{~s}, 3 \mathrm{H}), 1.49-1.35(\mathrm{~m}, 2 \mathrm{H}), 1.34-1.20(\mathrm{~m}, 14 \mathrm{H}), 0.88(\mathrm{t}, J=6.8 \mathrm{~Hz}, 3 \mathrm{H}) .{ }^{13} \mathrm{C}$ NMR (101 MHz, $\left.\mathrm{CDCl}_{3}\right) \delta 134.2(\mathrm{C}), 121.4(\mathrm{CH}), 51.9(\mathrm{CH}), 37.4\left(\mathrm{CH}_{2}\right), 36.4\left(\mathrm{CH}_{2}\right)$, $32.1\left(\mathrm{CH}_{2}\right), 29.9\left(\mathrm{CH}_{2}\right), 29.8\left(\mathrm{CH}_{2}\right), 29.7\left(\mathrm{CH}_{2}\right), 29.5\left(\mathrm{CH}_{2}\right), 26.4\left(\mathrm{CH}_{2}\right), 26.1\left(\mathrm{CH}_{3}\right)$, $22.8\left(\mathrm{CH}_{2}\right), 18.2\left(\mathrm{CH}_{3}\right), 14.3\left(\mathrm{CH}_{3}\right) . \mathrm{GC} t_{\mathrm{R}}=12.54 \mathrm{~min} . \mathrm{LRMS}(\mathrm{EI}) \mathrm{m} / z(\%) 225\left(\mathrm{M}^{+}\right.$, 0.1), 224 (0.3), 157 (13), 156 (100), 81 (5), 69 (6), 56 (10). HRMS (EI) calcd for $\mathrm{C}_{15} \mathrm{H}_{31} \mathrm{~N} 225.2457$, found 225.2469.

\section{(S)-1-Cyclohexyl-4-methyl-3-penten-1-amine (4c)}

Following the general procedure from Cyclohexylcarbaldehyde, compound $\mathbf{4 c}$ was obtained as a yellow oil after column chromatography (35 mg, $0.195 \mathrm{mmol}, 65 \%$ ): Enantiomeric excess was determined by HPLC of the corresponding benzamide (82:18 er on a Chiracel ODH column, n-Hexane/i-PrOH 95:5, $0.5 \mathrm{~mL} / \mathrm{min}, \lambda=220 \mathrm{~nm}$ ): major enantiomer $t_{\mathrm{R}}=20.8 \mathrm{~min}$, minor enantiomer $t_{\mathrm{R}}=18.5 \mathrm{~min}$. $[\alpha]^{20}{ }_{\mathrm{D}}-5.1\left(c 0.85, \mathrm{CH}_{2} \mathrm{Cl}_{2}\right)$. $\mathrm{R}_{\mathrm{f}}=0.20$ (9:1 $\left.\mathrm{CH}_{2} \mathrm{Cl}_{2} / \mathrm{MeOH}\right) . \mathrm{IR} v_{\max } / \mathrm{cm}^{-1} 3370,3292,2921,2851,1448,1376,890$, 817. ${ }^{1} \mathrm{H}$ NMR (400 MHz, $\left.\mathrm{CDCl}_{3}\right) \delta 5.15(\mathrm{t}, J=7.3 \mathrm{~Hz}, 1 \mathrm{H}), 2.65-2.49$ (m, 1H), $2.28-$ $2.10(\mathrm{~m}, 1 \mathrm{H}), 2.10-1.91(\mathrm{~m}, 2 \mathrm{H}), 1.77($ br d, $J=11.3 \mathrm{~Hz}, 3 \mathrm{H}), 1.73(\mathrm{~s}, 3 \mathrm{H}), 1.68$ (br s, 2H), $1.63(\mathrm{~s}, 3 \mathrm{H}), 1.38-0.91(\mathrm{~m}, 7 \mathrm{H}) .{ }^{13} \mathrm{C} \mathrm{NMR}\left(101 \mathrm{MHz}, \mathrm{CDCl}_{3}\right) \delta 134.1(\mathrm{C}), 121.9$ $(\mathrm{CH}), 56.7(\mathrm{CH}), 43.1(\mathrm{CH}), 33.1\left(\mathrm{CH}_{2}\right), 29.9\left(\mathrm{CH}_{2}\right), 28.4\left(\mathrm{CH}_{2}\right), 26.8\left(\mathrm{CH}_{2}\right), 26.7$ $\left(\mathrm{CH}_{2}\right), 26.5\left(\mathrm{CH}_{2}\right), 26.1\left(\mathrm{CH}_{3}\right), 18.2\left(\mathrm{CH}_{3}\right) . \mathrm{GC} t_{\mathrm{R}}=10.73 \mathrm{~min} . \quad$ LRMS (EI) $\mathrm{m} / z(\%)$ $181\left(\mathrm{M}^{+}, 0.1\right), 180$ (0.3), 113 (8), 112 (100), 98 (9), 95 (37), 81 (12), 67 (6), 55 (6). HRMS (EI) calcd for $\mathrm{C}_{12} \mathrm{H}_{23} \mathrm{~N} 181.1830$, found 181.1841 .

\section{$(5 R, 7 S)-2,7,11-T r i m e t h y l d o d e c a-2,10-d i e n-5-a m i n e ~(4 d)$}

Following the general procedure from $(S)$-Citronellal, compound $\mathbf{4 d}$ was obtained as a yellow oil after column chromatography (42 mg, $0.189 \mathrm{mmol}, 63 \%)$ : Diastereomeric excess was determined by comparing the ${ }^{13} \mathrm{C}$ NMR spectra of both diasteroisomers $4 \mathbf{d}$ and $4 \mathbf{e}\left(82: 18 \mathrm{dr}\right.$, see ESI). $[\alpha]^{20}{ }_{\mathrm{D}}+5.0\left(c\right.$ 1.07, $\left.\mathrm{CHCl}_{3}\right) . \mathrm{R}_{\mathrm{f}}=0.29\left(9: 1 \mathrm{CH}_{2} \mathrm{Cl}_{2} / \mathrm{MeOH}\right)$. IR $v_{\max } / \mathrm{cm}^{-1} 3350,2963,2913,2859,1727,1668,1450,1376,828,750 .{ }^{1} \mathrm{H}$ NMR (300 $\left.\mathrm{MHz} \mathrm{CDCl}_{3}\right) \delta 5.19-5.06(\mathrm{~m}, 2 \mathrm{H}), 2.87(\mathrm{tt}, J=9.6,5.0 \mathrm{~Hz}, 1 \mathrm{H}), 2.35-1.90(\mathrm{~m}, 6 \mathrm{H})$, $1.73(\mathrm{~d}, J=0.6 \mathrm{~Hz}, 3 \mathrm{H}), 1.68(\mathrm{~d}, J=1.0 \mathrm{~Hz}, 3 \mathrm{H}), 1.64(\mathrm{~s}, 3 \mathrm{H}), 1.60(\mathrm{~s}, 3 \mathrm{H}), 1.43-1.09$ $(\mathrm{m}, 5 \mathrm{H}), 0.89$ (d, $J=6.6 \mathrm{~Hz}, 3 \mathrm{H}) .{ }^{13} \mathrm{C} \mathrm{NMR}\left(101 \mathrm{MHz}, \mathrm{CDCl}_{3}\right) \delta 134.4(\mathrm{C}), 131.3(\mathrm{C})$, $124.9(\mathrm{CH}), 121.2(\mathrm{CH}), 49.4(\mathrm{CH}), 44.7\left(\mathrm{CH}_{2}\right), 37.9\left(\mathrm{CH}_{2}\right), 36.9\left(\mathrm{CH}_{2}\right), 29.4(\mathrm{CH})$, 
$26.1\left(\mathrm{CH}_{3}\right), 25.9\left(\mathrm{CH}_{3}\right), 25.6\left(\mathrm{CH}_{2}\right), 19.3\left(\mathrm{CH}_{3}\right), 18.2\left(\mathrm{CH}_{3}\right), 17.8\left(\mathrm{CH}_{3}\right) \cdot \mathrm{GC} \mathrm{t}_{\mathrm{R}}=12.04$ min. LRMS (EI) m/z (\%) $203\left(\mathrm{M}^{+}, 0.1\right), 155$ (12), 154 (100), 137 (19), 112 (6), 98 (7), 96 (5), 95 (38), 82 (89), 70 (13), 69 (39), 67 (10). HRMS (EI) calcd for $\mathrm{C}_{15} \mathrm{H}_{29} \mathrm{~N}$ 223.2300, found 223.2279.

\section{(5S,7S)-2,7,11-Trimethyldodeca-2,10-dien-5-amine (4e)}

Following the general procedure from $(S)$-Citronellal, but using ent-2b as chiral donor, compound 4e was obtained as a yellow oil after column chromatography (50 mg, 0.224 mmol, 75\%): Diastereomeric excess was determined by comparing the ${ }^{13} \mathrm{C} N M R$ spectra of both diasteroisomers $4 \mathbf{d}$ and $4 \mathbf{e}\left(93: 7 \mathrm{dr}\right.$, see ESI) $[\alpha]^{20}{ }_{\mathrm{D}}+10.6$ (c 0.80 , $\left.\mathrm{CH}_{2} \mathrm{Cl}_{2}\right) . \mathrm{R}_{\mathrm{f}}=0.27\left(9: 1 \mathrm{CH}_{2} \mathrm{Cl}_{2} / \mathrm{MeOH}\right) . \mathrm{IR} v_{\max } / \mathrm{cm}^{-1}$ 3351, 2963, 2913, 2848, 1727 , 1668, 1450, 1376, 829, 754. ${ }^{1} \mathrm{H}$ NMR $\left(300 \mathrm{MHz}, \mathrm{CDCl}_{3}\right) \delta 5.19-5.06(\mathrm{~m}, 2 \mathrm{H}), 2.88$ (dt, $J=12.0,7.4 \mathrm{~Hz}, 1 \mathrm{H}), 2.26$ (br s, 2H), $2.19-2.09(\mathrm{~m}, 1 \mathrm{H}), 2.06-1.90$ (m, 3H), 1.73 $(\mathrm{d}, J=0.6 \mathrm{~Hz}, 3 \mathrm{H}), 1.68(\mathrm{~d}, J=1.0 \mathrm{~Hz}, 3 \mathrm{H}), 1.64(\mathrm{~s}, 3 \mathrm{H}), 1.60(\mathrm{~s}, 3 \mathrm{H}), 1.58-1.51(\mathrm{~m}$, 1H), $1.44-1.30(\mathrm{~m}, 2 \mathrm{H}), 1.27-1.07(\mathrm{~m}, 2 \mathrm{H}), 0.91(\mathrm{~d}, J=6.6 \mathrm{~Hz}, 3 \mathrm{H}) .{ }^{13} \mathrm{C} \mathrm{NMR}(101$ $\left.\mathrm{MHz}, \mathrm{CDCl}_{3}\right) \delta 134.5(\mathrm{C}), 131.4(\mathrm{C}), 124.9(\mathrm{CH}), 121.1(\mathrm{CH}), 49.6(\mathrm{CH}), 44.9\left(\mathrm{CH}_{2}\right)$, $36.9\left(\mathrm{CH}_{2}\right), 36.0\left(\mathrm{CH}_{2}\right), 29.5(\mathrm{CH}), 26.1\left(\mathrm{CH}_{3}\right), 25.9\left(\mathrm{CH}_{3}\right), 25.4\left(\mathrm{CH}_{2}\right), 20.3\left(\mathrm{CH}_{3}\right)$, $18.2\left(\mathrm{CH}_{3}\right), 17.8\left(\mathrm{CH}_{3}\right) . \mathrm{GC} \mathrm{t}_{\mathrm{R}}=12.16 \mathrm{~min} . \mathrm{LRMS}(\mathrm{EI}) \mathrm{m} / \mathrm{z}(\%) 223\left(\mathrm{M}^{+}, 0.1\right), 155$ (12), 154 (100), 137 (19), 112 (6), 98 (7), 95 (38), 82 (9), 81 (89), 70 (13), 69 (39), 67 (10). HRMS (EI) calcd for $\mathrm{C}_{15} \mathrm{H}_{29} \mathrm{~N} 223.2300$, found 223.2283.

\section{(E,S)-6-Methyl-1-phenylhepta-1,5-dien-3-amine (4f)}

Following the general procedure from Cinnamaldehyde, compound $\mathbf{4 f}$ was obtained as a yellow oil after column chromatography (48 mg, $0.240 \mathrm{mmol}, 80 \%$ ): Enantiomeric excess was determined by HPLC of the corresponding benzamide (89:11 er on a Chiracel ODH column, n-Hexane/i-PrOH 95:5, $1 \mathrm{~mL} / \mathrm{min}, \quad \lambda=220 \mathrm{~nm}$ ): major enantiomer $t_{\mathrm{R}}=37.7 \mathrm{~min}$, minor enantiomer $t_{\mathrm{R}}=42.0 \mathrm{~min}$. $[\alpha]^{20}{ }_{\mathrm{D}}-8.5\left(c 0.92, \mathrm{CH}_{2} \mathrm{Cl}_{2}\right)$. $\mathrm{R}_{\mathrm{f}}=0.15\left(9: 1 \mathrm{CH}_{2} \mathrm{Cl}_{2} / \mathrm{MeOH}\right) . \mathrm{IR} v_{\max } / \mathrm{cm}^{-1}$ 3370, 3301, 2917, 2859, 1448, 1376, 812. ${ }^{1} \mathrm{H}$ NMR (400 MHz, $\left.\mathrm{CDCl}_{3}\right) \delta 7.42-7.29(\mathrm{~m}, 4 \mathrm{H}), 7.24-7.17(\mathrm{~m}, 1 \mathrm{H}), 6.51(\mathrm{~d}, J=$ $15.9 \mathrm{~Hz}, 1 \mathrm{H}), 6.21(\mathrm{dd}, J=15.9,6.8 \mathrm{~Hz}, 1 \mathrm{H}), 5.30-5.05(\mathrm{~m}, 1 \mathrm{H}), 3.53(\mathrm{q}, J=6.7 \mathrm{~Hz}$, 1H), $2.24(\mathrm{t}, J=7.0 \mathrm{~Hz}, 2 \mathrm{H}), 1.92$ (br s, 2H), 1.73 (s, 3H), 1.65 (s, 3H). ${ }^{13} \mathrm{C}$ NMR (101 $\left.\mathrm{MHz}, \mathrm{CDCl}_{3}\right) \delta 137.3(\mathrm{C}), 134.8(\mathrm{CH}), 134.3(\mathrm{C}), 129.1(\mathrm{CH}), 128.7(\mathrm{CH}), 127.4(\mathrm{CH})$, $126.4(\mathrm{CH}), 120.5(\mathrm{CH}), 54.3(\mathrm{CH}), 36.6\left(\mathrm{CH}_{2}\right), 26.1\left(\mathrm{CH}_{3}\right), 18.2\left(\mathrm{CH}_{3}\right) . \mathrm{GC} \mathrm{t}_{\mathrm{R}}=13.09$ 
min. LRMS (EI) m/z (\%) $201\left(\mathrm{M}^{+}, 0.1\right), 133$ (11), 132 (100), 130 (9), 117 (8), 115 (31). HRMS (EI) calcd for $\mathrm{C}_{14} \mathrm{H}_{19} \mathrm{~N} 201.1517$, found 201.1538.

\section{(S)-1-(2-Chlorophenyl)-4-methyl-3-penten-1-amine (4g)}

Following the general procedure from 2-Chlorobenzaldehyde, compound $\mathbf{4 g}$ was obtained as a yellow oil after column chromatography (32 mg, $0.156 \mathrm{mmol}, 52 \%$ ): Enantiomeric excess was determined by HPLC of the corresponding benzamide (92:8 er on a Chiracel ODH column, n-Hexane/i-PrOH 95:5, $1 \mathrm{~mL} / \mathrm{min}, \lambda=220 \mathrm{~nm}$ ): major enantiomer $t_{\mathrm{R}}=18.4 \mathrm{~min}$, minor enantiomer $t_{\mathrm{R}}=14.9 \mathrm{~min}$. $[\alpha]^{20}{ }_{\mathrm{D}}-69.8\left(\mathrm{c} 1.50, \mathrm{CHCl}_{3}\right)$. $\mathrm{R}_{\mathrm{f}}=0.50\left(9: 1 \mathrm{CH}_{2} \mathrm{Cl}_{2} / \mathrm{MeOH}\right) . \mathrm{IR} v_{\max } / \mathrm{cm}^{-1} 3370,3282,3060,2967,2913,2853,1668$, 1439, 1035, 854, 694. ${ }^{1} \mathrm{H}$ NMR $\left(300 \mathrm{MHz}, \mathrm{CDCl}_{3}\right) \delta 7.53(\mathrm{dd}, J=7.7,1.6 \mathrm{~Hz}, 1 \mathrm{H})$, $7.33(\mathrm{dd}, J=7.8,1.4 \mathrm{~Hz}, 1 \mathrm{H}), 7.29-7.24(\mathrm{~m}, 1 \mathrm{H}), 7.19-7.13(\mathrm{~m}, 1 \mathrm{H}), 5.17$ (tdd, $J=$ 6.7, 2.8, 1.4 Hz, 1H), $4.42(\mathrm{dd}, J=8.3,4.8 \mathrm{~Hz}, 1 \mathrm{H}), 2.44(\mathrm{dt}, J=11.6,5.7 \mathrm{~Hz}, 1 \mathrm{H}), 2.36$ $-2.19(\mathrm{~m}, 1 \mathrm{H}), 1.72$ (d, $J=0.7 \mathrm{~Hz}, 3 \mathrm{H}), 1.62$ (br s, 2H), 1.61 (s, 3H). ${ }^{13} \mathrm{C}$ NMR (101 $\left.\mathrm{MHz}, \mathrm{CDCl}_{3}\right) \delta 143.4(\mathrm{C}), 135.0(\mathrm{C}), 133.0(\mathrm{C}), 129.6(\mathrm{CH}), 127.9(\mathrm{CH}), 127.4(\mathrm{CH})$, $127.1(\mathrm{CH}), 120.8(\mathrm{CH}), 52.4(\mathrm{CH}), 36.4\left(\mathrm{CH}_{2}\right), 26.0\left(\mathrm{CH}_{3}\right), 18.1\left(\mathrm{CH}_{3}\right) . \mathrm{GC} \mathrm{t}_{\mathrm{R}}=11.89$ min. LRMS (EI) m/z (\%) 209 (0.1, M+), 142 (34), 141 (9), 140 (100), 115 (8), 113 (5, 78 (3, 77 (13). Anal. Calc'd for $\mathrm{C}_{12} \mathrm{H}_{16} \mathrm{ClN}$ : C 68.73, H 7.69, N 6.68. Found: C 68.99, H $7.52, \mathrm{~N} 6.58$.

\section{(S)-1-(3-Chlorophenyl)-4-methyl-3-penten-1-amine (4h)}

Following the general procedure from 3-Chlorobenzaldehyde, compound $\mathbf{4 h}$ was obtained as a yellow oil after column chromatography (27 mg, $0.129 \mathrm{mmol}, 45 \%$ ): Enantiomeric excess was determined by HPLC of the corresponding benzamide (89:11 er on a Chiracel ODH column, n-Hexane/i-PrOH 95:5, $1 \mathrm{~mL} / \mathrm{min}, \lambda=220 \mathrm{~nm}$ ): major enantiomer $t_{\mathrm{R}}=29.5 \mathrm{~min}$, minor enantiomer $t_{\mathrm{R}}=18.9 \mathrm{~min}$. $[\alpha]^{20}{ }_{\mathrm{D}}-30.2\left(c 0.87, \mathrm{CHCl}_{3}\right)$. $\mathrm{R}_{\mathrm{f}}=0.34$ (9:1 CH $\left.\mathrm{Cl}_{2} / \mathrm{MeOH}\right) . \mathrm{IR} v_{\max } / \mathrm{cm}^{-1} 3370,3281,2968,2913,1596,1573,1432$, 784, 697. ${ }^{1} \mathrm{H}$ NMR $\left(300 \mathrm{MHz}, \mathrm{CDCl}_{3}\right) \delta 7.36(\mathrm{t}, J=1.7 \mathrm{~Hz}, 1 \mathrm{H}), 7.26-7.16(\mathrm{~m}, 3 \mathrm{H})$, $5.19-4.97(\mathrm{~m}, 1 \mathrm{H}), 3.92(\mathrm{t}, J=6.7 \mathrm{~Hz}, 1 \mathrm{H}), 2.32(\mathrm{t}, J=7.0 \mathrm{~Hz}, 2 \mathrm{H}), 1.88(\mathrm{~d}, J=3.6$ $\mathrm{Hz}, 1 \mathrm{H}), 1.71$ (d, $J=1.0 \mathrm{~Hz}, 3 \mathrm{H}), 1.64$ (br s, $1 \mathrm{H}), 1.59$ (s, 3H). ${ }^{13} \mathrm{C}$ NMR (101 MHz, $\left.\mathrm{CDCl}_{3}\right) \delta 148.5(\mathrm{C}), 135.1(\mathrm{C}), 134.3(\mathrm{C}), 129.7(\mathrm{CH}), 127.1(\mathrm{CH}), 126.8(\mathrm{CH}), 124.8$ $(\mathrm{CH}), 120.6(\mathrm{CH}), 56.0(\mathrm{CH}), 38.5\left(\mathrm{CH}_{2}\right), 26.0\left(\mathrm{CH}_{3}\right), 18.1\left(\mathrm{CH}_{3}\right) . \mathrm{GC} \mathrm{t}_{\mathrm{R}}=12.24 \mathrm{~min}$. 
LRMS (EI) m/z (\%) 206 (1), 142 (32), 141 (9), 140 (100), 138 (3), 77 (13). HRMS $\left(\mathrm{ES}^{+}\right.$) calcd for $\mathrm{C}_{12} \mathrm{H}_{17} \mathrm{ClN} 210.1050$, found 210.1044 .

\section{(S)-1-(4-Chlorophenyl)-4-methyl-3-penten-1-amine (4i)}

Following the general procedure from 4-Chlorobenzaldehyde, compound $\mathbf{4 i}$ was obtained as a yellow oil after column chromatography (18 mg, $0.09 \mathrm{mmol}, 30 \%)$ : Enantiomeric excess was determined by HPLC of the corresponding benzamide (89:11 er on a Chiracel ODH column, n-Hexane/i-PrOH 95:5, $1 \mathrm{~mL} / \mathrm{min}, \lambda=220 \mathrm{~nm}$ ): major enantiomer $t_{\mathrm{R}}=32.8 \mathrm{~min}$, minor enantiomer $t_{\mathrm{R}}=21.4 \mathrm{~min}$. $[\alpha]^{20} \mathrm{D}-25.5\left(\mathrm{c} 1.50, \mathrm{CHCl}_{3}\right)$. $\mathrm{R}_{\mathrm{f}}=0.33\left(9: 1 \mathrm{CH}_{2} \mathrm{Cl}_{2} / \mathrm{MeOH}\right) . \mathrm{IR} v_{\max } / \mathrm{cm}^{-1} 3375,3286,2968,2912,1859,1668,1489$, 1089, 1013, 822. ${ }^{1} \mathrm{H}$ NMR (300 MHz, $\left.\mathrm{CDCl}_{3}\right) \delta 7.30(\mathrm{~s}, 4 \mathrm{H}), 5.10(\mathrm{tt}, J=7.3,1.4 \mathrm{~Hz}$, 1H), $3.95(\mathrm{t}, J=7.0 \mathrm{~Hz}, 1 \mathrm{H}), 2.32(\mathrm{t}, J=7.1 \mathrm{~Hz}, 2 \mathrm{H}), 1.72(\mathrm{~d}, J=0.9 \mathrm{~Hz}, 3 \mathrm{H}), 1.62(\mathrm{br}$ s, 2H), 1.60 (s, 3H). $\left.{ }^{13} \mathrm{C} \mathrm{NMR} \mathrm{(101} \mathrm{MHz,} \mathrm{CDCl}_{3}\right) \delta 144.7$ (C), 135.0 (C), 132.5 (C), $128.5(\mathrm{CH}), 127.9(\mathrm{CH}), 120.7(\mathrm{CH}), 55.8(\mathrm{CH}), 38.5\left(\mathrm{CH}_{2}\right), 26.0\left(\mathrm{CH}_{3}\right), 18.1\left(\mathrm{CH}_{3}\right)$. $\mathrm{GC} \mathrm{t}_{\mathrm{R}}=12.18 \mathrm{~min} . \mathrm{LRMS}(\mathrm{EI}) \mathrm{m} / z(\%) 143$ (9), 142 (34), 141 (28), 140 (100), 138 (8), 115 (8), 113 (21), 78 (6), 77 (36). HRMS (EI) calcd for $\mathrm{C}_{12} \mathrm{H}_{16} \mathrm{CIN}$ 209.0971, found 209.1251 .

\section{(S)-1-(3-Bromophenyl)-4-methyl-3-penten-1-amine (4j)}

Following the general procedure from 3-Bromobenzaldehyde, compound $\mathbf{4 j}$ was obtained as a yellow oil after column chromatography (37 mg, $0.146 \mathrm{mmol}, 50 \%$ ): Enantiomeric excess was determined by HPLC of the corresponding benzamide (89:11 er on a Chiracel ODH column, n-Hexane/i-PrOH 95:5, $1 \mathrm{~mL} / \mathrm{min}, \lambda=220 \mathrm{~nm}$ ): major enantiomer $t_{\mathrm{R}}=31.8 \mathrm{~min}$, minor enantiomer $t_{\mathrm{R}}=20.8 \mathrm{~min}$. $[\alpha]^{20} \mathrm{D}-22.6\left(c 1.20, \mathrm{CHCl}_{3}\right)$. $\mathrm{R}_{\mathrm{f}}=0.33$ (9:1 CH $\left.\mathrm{Cl}_{2} / \mathrm{MeOH}\right)$. IR $v_{\max } / \mathrm{cm}^{-1} 3370,3301,2966,2912,1593,1567,1427$, 1068, 782. ${ }^{1} \mathrm{H}$ NMR $\left(300 \mathrm{MHz}, \mathrm{CDCl}_{3}\right) \delta 7.51(\mathrm{t}, J=1.7 \mathrm{~Hz}, 1 \mathrm{H}), 7.39-7.33(\mathrm{~m}, 1 \mathrm{H})$, $7.29-7.23(\mathrm{~m}, 1 \mathrm{H}), 7.18(\mathrm{t}, J=7.7 \mathrm{~Hz}, 1 \mathrm{H}), 5.09(\mathrm{tdd}, J=6.0,2.8,1.4 \mathrm{~Hz}, 1 \mathrm{H}), 3.91$ (t, $J=6.7 \mathrm{~Hz}, 1 \mathrm{H}), 2.32(\mathrm{t}, J=7.0 \mathrm{~Hz}, 2 \mathrm{H}), 1.72($ br s, 2H), $1.70(\mathrm{~d}, J=0.9 \mathrm{~Hz}, 3 \mathrm{H})$, $1.58(\mathrm{~s}, 3 \mathrm{H}) .{ }^{13} \mathrm{C}$ NMR (101 MHz, $\left.\mathrm{CDCl}_{3}\right) \delta 148.7$ (C), 135.1 (C), 130.1 (C), 130.0 $(\mathrm{CH}), 129.7(\mathrm{CH}), 125.2(\mathrm{CH}), 122.6(\mathrm{CH}), 120.6(\mathrm{CH}), 55.9(\mathrm{CH}), 38.4\left(\mathrm{CH}_{2}\right), 26.0$ $\left(\mathrm{CH}_{3}\right), 18.1\left(\mathrm{CH}_{3}\right) . \mathrm{GC} \mathrm{t}_{\mathrm{R}}=12.87 \mathrm{~min}$. LRMS (EI) $\mathrm{m} / z(\%) 254$ (0.1), 187 (12), 186 (98), 185 (13), 184 (100), 182 (2), 157 (7), 104 (9), 77 (13). HRMS (ES ${ }^{+}$) calcd for $\mathrm{C}_{12} \mathrm{H}_{17} \mathrm{BrN} 254.0544$, found 254.0548. 


\section{(S)-1-(4-Nitrophenyl)-4-methyl-3-penten-1-amine (4k)}

Following the general procedure from 4-Nitrobenzaldehyde, compound $\mathbf{4 k}$ was obtained as a yellow oil after column chromatography (55 $\mathrm{mg}, 0.250 \mathrm{mmol}, 83 \%$ ): Enantiomeric excess was determined by HPLC of the corresponding benzamide (90:10 er on a Chiracel ODH column, n-Hexane/i-PrOH 90:10, $1 \mathrm{~mL} / \mathrm{min}, \lambda=220 \mathrm{~nm}$ ): major enantiomer $t_{\mathrm{R}}=36.5 \mathrm{~min}$, minor enantiomer $t_{\mathrm{R}}=30.1 \mathrm{~min} . \quad[\alpha]^{20}{ }_{\mathrm{D}}-21.7(c) 1.10$, $\left.\mathrm{CHCl}_{3}\right) . \mathrm{R}_{\mathrm{f}}=0.36\left(9: 1 \mathrm{CH}_{2} \mathrm{Cl}_{2} / \mathrm{MeOH}\right) . \mathrm{IR} v_{\max } / \mathrm{cm}^{-1} 3376,2971,2911,2848,1597$, 1507, 1342, 856, 751, 701. ${ }^{1} \mathrm{H}$ NMR $\left(300 \mathrm{MHz}, \mathrm{CDCl}_{3}\right) \delta 8.19(\mathrm{dd}, J=8.8,2.1 \mathrm{~Hz}$, 2H), $7.54(\mathrm{dd}, J=8.6,2.1 \mathrm{~Hz}, 2 \mathrm{H}), 5.08(\mathrm{tt}, J=7.4,1.4 \mathrm{~Hz}, 1 \mathrm{H}), 4.08(\mathrm{t}, J=6.7 \mathrm{~Hz}$, $1 \mathrm{H}), 2.34(\mathrm{t}, J=7.1 \mathrm{~Hz}, 2 \mathrm{H}), 1.71(\mathrm{~d}, J=0.7 \mathrm{~Hz}, 3 \mathrm{H}), 1.64(\mathrm{br} \mathrm{s}, 2 \mathrm{H}), 1.57(\mathrm{~s}, 3 \mathrm{H}) .{ }^{13} \mathrm{C}$ NMR (101 MHz, $\left.\mathrm{CDCl}_{3}\right) \delta 153.8(\mathrm{C}), 147.0(\mathrm{C}), 135.8(\mathrm{C}), 127.4(\mathrm{CH}), 127.4(\mathrm{CH})$, $123.7(\mathrm{CH}), 123.7(\mathrm{CH}), 119.9(\mathrm{CH}), 55.9(\mathrm{CH}), 38.5\left(\mathrm{CH}_{2}\right), 26.0\left(\mathrm{CH}_{3}\right), 18.1\left(\mathrm{CH}_{3}\right)$. $\mathrm{GC} \mathrm{t}_{\mathrm{R}}=14.37 \mathrm{~min} . \mathrm{LRMS}(\mathrm{EI}) \mathrm{m} / z$ (\%) 152 (9), 151 (100), 121 (4), 105 (31), 104 (13), 93 (5), 78 (5), 77 (6). HRMS (EI) calcd for $\mathrm{C}_{12} \mathrm{H}_{16} \mathrm{~N}_{2} \mathrm{O}_{2} 220.1212$, found 220.1202.

\section{(S)-1-(5-Fluoro-2-nitrophenyl)-4-methyl-3-penten-1-amine (4l)}

Following the general procedure from 5-Fluoro-2-nitrobenzaldehyde, compound $\mathbf{4 l}$ was obtained as a yellow oil after column chromatography (42 mg, $0.176 \mathrm{mmol}, 60 \%$ ): Enantiomeric excess was determined by HPLC of the corresponding benzamide (94:6 er on a Chiracel ODH column, n-Hexane/i-PrOH 95:5, $1 \mathrm{~mL} / \mathrm{min}, \lambda=220 \mathrm{~nm}$ ): major enantiomer $t_{\mathrm{R}}=37.8 \mathrm{~min}$, minor enantiomer $t_{\mathrm{R}}=28.0 \mathrm{~min}$. $[\alpha]^{20}{ }_{\mathrm{D}}-89.8\left(c 0.75, \mathrm{CHCl}_{3}\right)$. $\mathrm{R}_{\mathrm{f}}=0.53$ (9:1 CH $\left.\mathrm{Cl}_{2} / \mathrm{MeOH}\right) . \mathrm{IR} v_{\max } / \mathrm{cm}^{-1} 3390,3302,2970,2916,2849,1584,1512$, 1352, 851, 757, 704. ${ }^{1} \mathrm{H} \mathrm{NMR}\left(400 \mathrm{MHz}, \mathrm{CDCl}_{3}\right) \delta 7.88(\mathrm{dd}, J=9.0,5.1 \mathrm{~Hz}, 1 \mathrm{H}), 7.59$ $(\mathrm{dd}, J=9.9,2.8 \mathrm{~Hz}, 1 \mathrm{H}), 7.03(\mathrm{ddd}, J=9.0,7.1,2.8 \mathrm{~Hz}, 1 \mathrm{H}), 5.13(\mathrm{ttd}, J=6.6,2.7,1.3$ $\mathrm{Hz}, 1 \mathrm{H}), 4.59$ (ddd, $J=8.2,4.8,1.6 \mathrm{~Hz}, 1 \mathrm{H}), 2.47-2.36(\mathrm{~m}, 1 \mathrm{H}), 2.34-2.23(\mathrm{~m}, 1 \mathrm{H})$, 1.72 (s, 3H), 1.60 (br s, 5H). ${ }^{13} \mathrm{C}$ NMR (101 MHz, $\left.\mathrm{CDCl}_{3}\right) \delta 165.1$ (d, J=255.3 Hz, C), 145.3 (d, $J=8.0 \mathrm{~Hz}, \mathrm{C}), 145.2$ (C), 136.1 (C), 127.1 (d, $J=9.5 \mathrm{~Hz}, \mathrm{CH}), 120.0$ (CH), $115.5(\mathrm{~d}, J=24.4 \mathrm{~Hz}, \mathrm{CH}), 114.7(\mathrm{~d}, J=24.4 \mathrm{~Hz}, \mathrm{CH}), 50.7(\mathrm{CH}), 37.5\left(\mathrm{CH}_{2}\right), 26.0$ $\left(\mathrm{CH}_{3}\right), 18.1\left(\mathrm{CH}_{3}\right) . \mathrm{GC} \mathrm{t}_{\mathrm{R}}=13.04 \mathrm{~min} . \mathrm{LRMS}(\mathrm{EI}) \mathrm{m} / \mathrm{z}$ (\%) 170 (9), 169 (100), 152 (3), 123 (17), 122 (17), 122 (23), 69 (4). HRMS (EI) calcd for $\mathrm{C}_{12} \mathrm{H}_{16} \mathrm{~N}_{2} \mathrm{O}_{2} \mathrm{~F}$ 239.1196, found 239.1197. 


\section{(R)-5-Prenyl-2-pyrrolidinone (4m)}

Following the general procedure from Methyl 4-oxobutanoate, ${ }^{22}$ compound $\mathbf{4 m}$ was obtained as a yellow oil after column chromatography (35 mg, $0.225 \mathrm{mmol}, 75 \%$ ): Enantiomeric excess was directly determined by HPLC of the lactam (88:12 er on a Chiracel ODH column, n-Hexane/i-PrOH 90:10, $1 \mathrm{~mL} / \mathrm{min}, \lambda=220 \mathrm{~nm}$ ): major enantiomer $t_{\mathrm{R}}=8.1 \mathrm{~min}$, minor enantiomer $t_{\mathrm{R}}=9.5 \mathrm{~min}$. $[\alpha]^{20}{ }_{\mathrm{D}}-14.5\left(c 0.90, \mathrm{CHCl}_{3}\right)$. $\mathrm{R}_{\mathrm{f}}=0.20$ (1:3 Hexane:EtOAc). IR $v_{\max } / \mathrm{cm}^{-1}$ 3210, 3100, 2969, 2915, 1689, 1440, 1424, 1343, 856. ${ }^{1} \mathrm{H}$ NMR (300 MHz, $\left.\mathrm{CDCl}_{3}\right) \delta 6.05$ (br s, $\left.1 \mathrm{H}\right), 5.09$ (tdd, $J=6.9,2.8$, $1.4 \mathrm{~Hz}, 1 \mathrm{H}), 3.65$ (p, $J=6.6 \mathrm{~Hz}, 1 \mathrm{H}), 2.38-2.29(\mathrm{~m}, 2 \mathrm{H}), 2.28-2.14(\mathrm{~m}, 3 \mathrm{H}), 1.82-$ $1.74(\mathrm{~m}, 1 \mathrm{H}), 1.72(\mathrm{~s}, 3 \mathrm{H}), 1.63(\mathrm{~s}, 3 \mathrm{H}) .{ }^{13} \mathrm{C} \mathrm{NMR}\left(101 \mathrm{MHz}, \mathrm{CDCl}_{3}\right) \delta 178.1(\mathrm{C})$, $135.5(\mathrm{C}), 119.3(\mathrm{CH}), 54.7(\mathrm{CH}), 35.3\left(\mathrm{CH}_{2}\right), 30.3\left(\mathrm{CH}_{2}\right), 26.9\left(\mathrm{CH}_{2}\right), 26.0\left(\mathrm{CH}_{3}\right), 18.1$ $\left(\mathrm{CH}_{3}\right) . \mathrm{GC} \mathrm{t}_{\mathrm{R}}=11.30 \mathrm{~min}$. LRMS (EI) $\mathrm{m} / z(\%) 153\left(\mathrm{M}^{+}, 2\right), 85(5), 84$ (100), 69 (2), 56 (6), 55 (3). HRMS (ES ${ }^{+}$) calcd for $\mathrm{C}_{9} \mathrm{H}_{16} \mathrm{NO}$ 154.1232, found 154.1231.

\section{(S)-5-Prenyl-2-pyrrolidinone (ent-4m)}

Following the general procedure from Methyl 4-oxobutanoate, compound ent-4m was obtained with identical spectroscopic and physical data as compound $\mathbf{4 m}$ except for $[\alpha]^{20}{ }_{D}+14.1\left(c 0.95, \mathrm{CHCl}_{3}\right)$. In this case ent-2b was used as chiral donor.

\section{(R)-Ethyl 6-amino-9-methyl-8-decenoate (4n)}

Following the general procedure from Ethyl 6-oxohexanoate, ${ }^{23}$ but in a larger scale (1.45 mmol of donor $\mathbf{2 b}$ ), compound $\mathbf{4 n}$ was obtained as a yellow oil after column chromatography (286 mg, $1.261 \mathrm{mmol}, 87 \%$ ): Enantiomeric excess was determined by HPLC of the corresponding benzamide (85:15 er on a Chiracel ODH column, nHexane/i-PrOH 95:5, $1 \mathrm{~mL} / \mathrm{min}, \lambda=220 \mathrm{~nm}$ ): major enantiomer $t_{\mathrm{R}}=20.5 \mathrm{~min}$, minor enantiomer $t_{\mathrm{R}}=30.1 \mathrm{~min}$. $[\alpha]_{\mathrm{D}}^{20}-5.3$ (c 1.20, $\left.\mathrm{CHCl}_{3}\right)$. $\mathrm{R}_{\mathrm{f}}=0.30$ (1:3 Hexane:EtOAc). IR $v_{\max } / \mathrm{cm}^{-1} 3439,3370,2972,2928,2860,1733,1248,1179,851,722 .{ }^{1} \mathrm{H}$ NMR (300 $\left.\mathrm{MHz}, \mathrm{CDCl}_{3}\right) \delta 5.13(\mathrm{dd}, J=7.9,6.9 \mathrm{~Hz}, 1 \mathrm{H}), 4.12(\mathrm{q}, J=7.1 \mathrm{~Hz}, 2 \mathrm{H}), 2.77(\mathrm{br} \mathrm{s}, 1 \mathrm{H})$, $2.31(\mathrm{t}, J=7.4 \mathrm{~Hz}, 2 \mathrm{H}), 2.23$ (br s, 2H), $2.18-1.94(\mathrm{~m}, 2 \mathrm{H}), 1.73$ (s, 3H), $1.70-1.65$ $(\mathrm{m}, 1 \mathrm{H}), 1.63(\mathrm{~s}, 3 \mathrm{H}), 1.62-1.54(\mathrm{~m}, J=13.0 \mathrm{~Hz}, 1 \mathrm{H}), 1.55-1.40(\mathrm{~m}, 2 \mathrm{H}), 1.40-$ $1.30(\mathrm{~m}, 2 \mathrm{H}), 1.26(\mathrm{t}, J=7.1 \mathrm{~Hz}, 3 \mathrm{H}) .{ }^{13} \mathrm{C} \mathrm{NMR}\left(101 \mathrm{MHz}, \mathrm{CDCl}_{3}\right) \delta 173.8(\mathrm{C}), 134.5$ (C), $121.1(\mathrm{CH}), 60.3\left(\mathrm{CH}_{2}\right), 51.7(\mathrm{CH}), 36.8\left(\mathrm{CH}_{2}\right), 36.3\left(\mathrm{CH}_{2}\right), 34.4\left(\mathrm{CH}_{2}\right), 26.03$ $\left(\mathrm{CH}_{3}\right), 25.9\left(\mathrm{CH}_{2}\right), 25.1\left(\mathrm{CH}_{2}\right), 18.2\left(\mathrm{CH}_{3}\right), 14.4\left(\mathrm{CH}_{3}\right) . \mathrm{GC} t_{\mathrm{R}}=12.46 \min$. LRMS (EI) 
m/z (\%) $227\left(\mathrm{M}^{+}, 0.1\right), 182$ (9), 159 (9), 158 (100), 140 (13), 113 (8), 112 (72), 84 (17), 81 (12), 69 (27), 67 (17), 56 (23). Anal. Calc'd for $\mathrm{C}_{13} \mathrm{H}_{25} \mathrm{NO}_{2}$ : C 68.68, H 11.08, N 6.16. Found: C 68.30, H 10.71, N 5.79.

\section{(S)-Ethyl 6-amino-9-methyl-8-decenoate (ent-4n)}

Following the general procedure from Ethyl 6-oxohexanoate, compound ent-4n was obtained with identical spectroscopic and physical data as compound $4 \mathbf{n}$ except for $[\alpha]^{20}{ }_{\mathrm{D}}+5.5\left(c 0.95, \mathrm{CHCl}_{3}\right)$. In this case ent-2b was used as chiral donor.

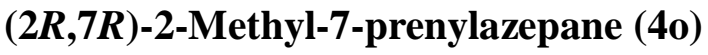

Following the general procedure from 6-Oxoheptanal. ${ }^{24}$ However, instead of adding $\mathrm{HONH}_{2} \cdot \mathrm{AcOH}$ to the arranged mixture, $\mathrm{NaBH}_{3} \mathrm{CN}(1.8 \mathrm{mmol}, 114 \mathrm{mg})$ was added and the reaction was stirred for $12 \mathrm{~h}$ at room temperature. Then, the reaction mixture was diluted with EtOAc $(5 \mathrm{~mL})$ and $\mathrm{NaOH}(2 \mathrm{M}, 5 \mathrm{~mL})$, and extracted with EtOAc $(3 \mathrm{x}$ $5 \mathrm{~mL})$, washed with $\mathrm{NaOH}(2 \mathrm{M}, 5 \mathrm{~mL})$ and brine $(5 \mathrm{~mL})$. Organics were dried over $\mathrm{MgSO}_{4}$, filtered and concentrated under reduced pressure. The residue was purified by column chromatography (gradient from 97:3 $\mathrm{CH}_{2} \mathrm{Cl}_{2} / \mathrm{MeOH}$ to 90:10:0.05 $\left.\mathrm{CH}_{2} \mathrm{Cl}_{2} / \mathrm{MeOH} / 20 \% \mathrm{NH}_{4} \mathrm{OH}\right)$ to provide the desired free amine $4 \mathbf{o}$ as a yellow oil (20 $\mathrm{mg}, 0.110 \mathrm{mmol}, 37 \%)$ : Enantiomeric excess was determined by HPLC of the corresponding benzamide (86:14 er on a Chiracel ODH column, n-Hexane/i-PrOH 98:2, $1 \mathrm{~mL} / \mathrm{min}, \lambda=220 \mathrm{~nm}$ ): major enantiomer $t_{\mathrm{R}}=17.0 \mathrm{~min}$, minor enantiomer $t_{\mathrm{R}}=20.8$ min. $[\alpha]^{20}-1.5\left(c 1.10, \mathrm{CHCl}_{3}\right) . \mathrm{R}_{\mathrm{f}}=0.37$ (9:1 $\left.\mathrm{CH}_{2} \mathrm{Cl}_{2} / \mathrm{MeOH}\right) . \mathrm{IR} v_{\max } / \mathrm{cm}^{-1} 3311$, 2962, 2924, 2855, 1678, 1450, 1375, 751. ${ }^{1} \mathrm{H}$ NMR (300 MHz, $\left.\mathrm{CDCl}_{3}\right) \delta 5.15-5.04$ (m, 1H), $2.82(\mathrm{dqd}, J=9.6,6.4,3.3 \mathrm{~Hz}, 1 \mathrm{H}), 2.67$ (ddd, $J=10.0,7.2,3.2 \mathrm{~Hz}, 1 \mathrm{H}), 2.24$ $-2.02(\mathrm{~m}, 2 \mathrm{H}), 1.78-1.73(\mathrm{~m}, 2 \mathrm{H}), 1.72(\mathrm{~d}, J=0.9 \mathrm{~Hz}, 3 \mathrm{H}), 1.71-1.64(\mathrm{~m}, 3 \mathrm{H}), 1.62$ (s, 3H), $1.61-1.50(\mathrm{~m}, 2 \mathrm{H}), 1.49-1.36(\mathrm{~m}, 2 \mathrm{H}), 1.14(\mathrm{~d}, J=6.4 \mathrm{~Hz}, 3 \mathrm{H}) .{ }^{13} \mathrm{C} \mathrm{NMR}$ $\left(101 \mathrm{MHz}, \mathrm{CDCl}_{3}\right) \delta 134.4(\mathrm{C}), 121.5(\mathrm{CH}), 59.7(\mathrm{CH}), 55.2(\mathrm{CH}), 38.0\left(\mathrm{CH}_{2}\right), 35.9$ $\left(\mathrm{CH}_{2}\right), 35.7\left(\mathrm{CH}_{2}\right), 25.8\left(\mathrm{CH}_{3}\right), 25.3\left(\mathrm{CH}_{2}\right), 25.0\left(\mathrm{CH}_{2}\right), 23.6\left(\mathrm{CH}_{3}\right), 18.0\left(\mathrm{CH}_{3}\right) \cdot \mathrm{GC} \mathrm{t}_{\mathrm{R}}=$ 9.19 min. LRMS (EI) m/z (\%) $181\left(\mathrm{M}^{+}, 1\right), 113$ (8), 112 (100), 95 (10), 82 (5), 69 (7), 56 (6). HRMS (ES ${ }^{+}$) calcd for $\mathrm{C}_{12} \mathrm{H}_{24} \mathrm{~N} 182.1909$, found 182.1906. 
Following the general procedure from 6-Oxoheptanal, compound ent-4o was obtained with identical spectroscopic and physical data as compound 4o except for $[\alpha]^{20}{ }_{\mathrm{D}}+1.8(c$ $\left.0.95, \mathrm{CHCl}_{3}\right)$. In this case ent-2b was used as chiral donor.

\section{General procedure for the synthesis of pyrrolidines 5a and $5 n$.}

To a solution of free amine $4 \mathbf{a}$ or $\mathbf{4 n}(1.17 \mathrm{mmol})$ in $\mathrm{CH}_{2} \mathrm{Cl}_{2}(11 \mathrm{~mL})$ at $0{ }^{\circ} \mathrm{C}$, were added $\mathrm{Et}_{3} \mathrm{~N}(248 \mu \mathrm{L}, 1.75 \mathrm{mmol})$ and $p$-nitrobenzenesulfonyl chloride (310 mg, 1.40 mmol). The solution was allowed to warm to $25^{\circ} \mathrm{C}$ and stirred over $12 \mathrm{~h}$. The reaction was quenched with $\mathrm{HCl}(1 \mathrm{M})$, and after phase separation, the organic layer was washed with $\mathrm{NaOH}(1 \mathrm{M})$, followed by water and brine. Evaporation of the solvent under vacuum gave crude $\mathbf{4 a - N s}$ or $\mathbf{4 n}-\mathrm{Ns}$, which were purified by column chromatography (9:1 Hexane/EtOAc) and totally characterized (see physical and spectroscopical data of the $p$-nosylate derivatives below). At this point, trifluoromethanesulfonic acid (19 $\mu \mathrm{L}$, $0.2 \mathbf{m m o l})$ was added to a solution of $\mathbf{4 a}-\mathrm{Ns}$ or $\mathbf{4 n}-\mathrm{Ns}$ in dry toluene $(70 \mathrm{~mL})$ and the reaction mixture was stirred at $0{ }^{\circ} \mathrm{C}$ for $1 \mathrm{~h}$. After concentration under reduced pressure, the residue was purified by column chromatography (9:1 Hexane/EtOAc) to provide the desired pyrrolidines $\mathbf{5 a}$ or $\mathbf{5 n}$.

\section{(R)-p-Nosylate of $4 \mathrm{a}(4 \mathrm{a}-\mathrm{Ns})$}

Compound 4a-Ns was obtained according to the general procedure as a white solid (392 $\mathrm{mg}, 1.01 \mathrm{mmol}, 86 \%)$ : $\mathrm{mp} 72.8-75.2^{\circ} \mathrm{C}$. $[\alpha]^{20}{ }_{\mathrm{D}}-8.6\left(c 0.95, \mathrm{CHCl}_{3}\right) . \mathrm{R}_{\mathrm{f}}=0.15(9: 1$ Hexane/EtOAc). IR $v_{\max } / \mathrm{cm}^{-1} 3289,3027,2926,2859,1605,1528,1347,1308,1161$, 1092, 852, 735. ${ }^{1} \mathrm{H}$ NMR $\left(300 \mathrm{MHz}, \mathrm{CDCl}_{3}\right) \delta 8.31(\mathrm{~d}, J=9.0 \mathrm{~Hz}, 2 \mathrm{H}), 7.98(\mathrm{~d}, J=8.9$ $\mathrm{Hz}, 2 \mathrm{H}), 7.29-7.27(\mathrm{~m}, 1 \mathrm{H}), 7.25-7.23(\mathrm{~m}, 1 \mathrm{H}), 7.23-7.16(\mathrm{~m}, 1 \mathrm{H}), 7.09-7.05$ (m, 2H), 4.84 (ddd, $J=7.5,4.4,1.3 \mathrm{~Hz}, 1 \mathrm{H}), 4.56$ (br s, 1H), $3.41-3.26$ (m, 1H), 2.67 $2.50(\mathrm{~m}, 2 \mathrm{H}), 2.18(\mathrm{dt}, J=13.6,6.7 \mathrm{~Hz}, 1 \mathrm{H}), 2.13-2.05(\mathrm{~m}, 1 \mathrm{H}), 1.88-1.78(\mathrm{~m}, 1 \mathrm{H})$, 1.73 (ddt, $J=14.0,9.3,7.0 \mathrm{~Hz}, 1 \mathrm{H}), 1.61(\mathrm{~d}, J=0.6 \mathrm{~Hz}, 3 \mathrm{H}), 1.51(\mathrm{~s}, 3 \mathrm{H}) .{ }^{13} \mathrm{C} \mathrm{NMR}$ $\left(101 \mathrm{MHz}, \mathrm{CDCl}_{3}\right) \delta 150.0(\mathrm{C}), 147.0(\mathrm{C}), 141.0(\mathrm{C}), 136.5(\mathrm{C}), 128.7(\mathrm{CH}), 128.4$ $(\mathrm{CH}), 128.4(\mathrm{CH}), 126.3(\mathrm{CH}), 124.4(\mathrm{CH}), 118.2(\mathrm{CH}), 54.4(\mathrm{CH}), 36.6\left(\mathrm{CH}_{2}\right), 33.4$ $\left(\mathrm{CH}_{2}\right), 31.9\left(\mathrm{CH}_{2}\right), 26.1\left(\mathrm{CH}_{3}\right), 18.1\left(\mathrm{CH}_{3}\right) . \mathrm{GC} \mathrm{t}_{\mathrm{R}}=24.32 \mathrm{~min} . \mathrm{LRMS}(\mathrm{EI}) \mathrm{m} / z(\%) 320$ (4), 319 (22), 289 (4), 156 (10), 132 (5), 122 (5), 118 (10), 117 (100), 92 (12), 91 (93), 77 (4), 65 (5). HRMS (ES ${ }^{+}$) calcd for $\mathrm{C}_{20} \mathrm{H}_{25} \mathrm{~N}_{2} \mathrm{O}_{4} \mathrm{~S} 389.1535$, found 389.1539. 


\section{$(R)$-p-Nosylate of $4 n(4 n-N s)$}

Compound 4n-Ns was obtained according to the general procedure as a yellow wax (420 mg, 1.02 mmol, 88\%): $[\alpha]^{20}{ }_{\mathrm{D}}+6.0\left(c 0.95, \mathrm{CHCl}_{3}\right) . \mathrm{R}_{\mathrm{f}}=0.28(8: 2 \mathrm{Hexane} / \mathrm{EtOAc})$. IR $v_{\max } / \mathrm{cm}^{-1} 3280,2931,2863,1730,1529,1348,1307,1160,1091,853,735 .{ }^{1} \mathrm{H}$ NMR (300 MHz, $\left.\mathrm{CDCl}_{3}\right) \delta 8.35(\mathrm{~d}, J=9.0 \mathrm{~Hz}, 2 \mathrm{H}), 8.04(\mathrm{~d}, J=9.0 \mathrm{~Hz}, 2 \mathrm{H}), 4.83$ (ddd, $J=7.5,6.1,1.4 \mathrm{~Hz}, 1 \mathrm{H}), 4.54(\mathrm{~d}, J=8.4 \mathrm{~Hz}, 1 \mathrm{H}), 4.11(\mathrm{q}, J=7.1 \mathrm{~Hz}, 2 \mathrm{H}), 3.38-3.23$ (m, 1H), 2.22 (t, $J=7.4 \mathrm{~Hz}, 2 \mathrm{H}), 2.18-1.94(\mathrm{~m}, 2 \mathrm{H}), 1.60(\mathrm{~s}, 3 \mathrm{H}), 1.55(\mathrm{~d}, J=7.5 \mathrm{~Hz}$, $2 \mathrm{H}), 1.50(\mathrm{~s}, 3 \mathrm{H}), 1.49-1.28(\mathrm{~m}, 3 \mathrm{H}), 1.25(\mathrm{t}, J=7.1 \mathrm{~Hz}, 3 \mathrm{H}), 1.22-1.13(\mathrm{~m}, 1 \mathrm{H}) .{ }^{13} \mathrm{C}$ NMR (101 MHz, $\left.\mathrm{CDCl}_{3}\right) \delta 173.6(\mathrm{C}), 150.1$ (C), $147.3(\mathrm{C}), 136.2(\mathrm{C}), 128.4(\mathrm{CH})$, $124.4(\mathrm{CH}), 118.4(\mathrm{CH}), 60.5\left(\mathrm{CH}_{2}\right), 54.8(\mathrm{CH}), 34.8\left(\mathrm{CH}_{2}\right), 34.1\left(\mathrm{CH}_{2}\right), 33.6\left(\mathrm{CH}_{2}\right)$, $26.0\left(\mathrm{CH}_{3}\right), 25.2\left(\mathrm{CH}_{2}\right), 24.7\left(\mathrm{CH}_{2}\right), 18.1\left(\mathrm{CH}_{3}\right), 14.4\left(\mathrm{CH}_{3}\right) . \mathrm{GC} \mathrm{t}_{\mathrm{R}}=25.88 \mathrm{~min}$. LRMS (EI) m/z (\%) 344 (6), 343 (36), 313 (8), 299 (9), 298 (16), 297 (100), 281 (9), 269 (11), 207 (24), 186 (6), 157 (5), 156 (17), 122 (13), 95 (28), 69 (71). HRMS (ES ${ }^{+}$) calcd for $\mathrm{C}_{19} \mathrm{H}_{29} \mathrm{~N}_{2} \mathrm{O}_{6} \mathrm{~S} 413.1746$, found 413.1732 .

\section{(R)-2,2-dimethyl-5-phenethyl-1-(p-nosyl)pyrrolidine (5a)}

Compound 5a was obtained according to the general procedure as a white solid (361 mg, 0.93 mmol, 93\%): mp $86.8-89.5{ }^{\circ} \mathrm{C} .[\alpha]^{20}{ }_{\mathrm{D}}-59.4\left(c 0.95, \mathrm{CHCl}_{3}\right) . \mathrm{R}_{\mathrm{f}}=0.30(9: 1$ Hexane/EtOAc). IR $v_{\max } / \mathrm{cm}^{-1}$ 2959, 29223, 2859, 1525, 1455, 1342, 1151, 855, 738. ${ }^{1} \mathrm{H}$ NMR $\left(300 \mathrm{MHz}, \mathrm{CDCl}_{3}\right) \delta 8.20(\mathrm{~d}, J=8.8 \mathrm{~Hz}, 2 \mathrm{H}), 7.76(\mathrm{~d}, J=8.8 \mathrm{~Hz}, 2 \mathrm{H}), 7.36-$ $7.30(\mathrm{~m}, 2 \mathrm{H}), 7.30-7.26(\mathrm{~m}, 0.7 \mathrm{H}), 7.26-7.23(\mathrm{~m}, 0.3 \mathrm{H}), 7.21-7.14(\mathrm{~m}, 2 \mathrm{H}), 3.68-$ $3.57(\mathrm{~m}, 1 \mathrm{H}), 2.72(\mathrm{ddd}, J=13.4,8.5,4.6 \mathrm{~Hz}, 1 \mathrm{H}), 2.50(\mathrm{dt}, J=13.9,8.4 \mathrm{~Hz}, 1 \mathrm{H}), 2.33$ $-2.22(\mathrm{~m}, 1 \mathrm{H}), 2.07-1.93(\mathrm{~m}, 1 \mathrm{H}), 1.85-1.69(\mathrm{~m}, 4 \mathrm{H}), 1.60(\mathrm{~s}, 3 \mathrm{H}), 1.31(\mathrm{~s}, 3 \mathrm{H}) .{ }^{13} \mathrm{C}$ NMR (101 MHz, $\left.\mathrm{CDCl}_{3}\right) \delta 149.6(\mathrm{C}), 147.6(\mathrm{C}), 141.0(\mathrm{C}), 128.8(\mathrm{CH}), 128.7(\mathrm{CH})$, $128.7(\mathrm{CH}), 126.3(\mathrm{CH}), 124.1(\mathrm{CH}), 67.1(\mathrm{C}), 61.4(\mathrm{CH}), 40.6\left(\mathrm{CH}_{2}\right), 36.6\left(\mathrm{CH}_{2}\right), 32.9$ $\left(\mathrm{CH}_{2}\right), 31.5\left(\mathrm{CH}_{3}\right), 27.1\left(\mathrm{CH}_{2}\right), 26.6\left(\mathrm{CH}_{3}\right) . \mathrm{GC} \mathrm{t} \mathrm{R}_{2}=24.01 \mathrm{~min}$. LRMS (EI) $\mathrm{m} / z(\%) 373$ (5), 15 (100), 253 (5), 156 (5), 122 (12), 117 (6), 105 (5), 98 (14), 97 (8), 96 (6), 91 (25), 83 (6), 82 (17), 81 (82), 65 (4). HRMS (ES ${ }^{+}$) calcd for $\mathrm{C}_{20} \mathrm{H}_{25} \mathrm{~N}_{2} \mathrm{O}_{4} \mathrm{~S} 389.1535$, found 389.1538 .

\section{(R)-Ethyl 5-(5,5-dimethyl-1-(p-nosyl)pyrrolidin-2-yl)pentanoate (5n)}

Compound 5n was obtained according to the general procedure as a yellow oil (380 $\mathrm{mg}$, 0.92 mmol, 93\%): $[\alpha]^{20}{ }_{D}-29.3\left(c 0.95, \mathrm{CHCl}_{3}\right) . \mathrm{R}_{\mathrm{f}}=0.40$ (8:2 Hexane/EtOAc). IR $v_{\max } /$ 
$\mathrm{cm}^{-1} 3110,2970,2939,2869,1731,1528,1462,1347,1305,1152,1092,854,736 .{ }^{1} \mathrm{H}$ $\operatorname{NMR}\left(300 \mathrm{MHz}, \mathrm{CDCl}_{3}\right) \delta 8.33(\mathrm{~d}, J=9.0 \mathrm{~Hz}, 2 \mathrm{H}), 8.05(\mathrm{~d}, J=9.1 \mathrm{~Hz}, 2 \mathrm{H}), 4.13(\mathrm{q}, J$ $=7.1 \mathrm{~Hz}, 2 \mathrm{H}), 3.80(\mathrm{ddd}, J=9.4,6.6,2.9 \mathrm{~Hz}, 1 \mathrm{H}), 2.29$ (t, $J=7.4 \mathrm{~Hz}, 2 \mathrm{H}), 2.07-1.59$ (m, 7H), $1.58(\mathrm{~s}, 3 \mathrm{H}), 1.57-1.42(\mathrm{~m}, 2 \mathrm{H}), 1.36-1.32(\mathrm{~m}, 1 \mathrm{H}), 1.31(\mathrm{~s}, 3 \mathrm{H}), 1.26(\mathrm{t}, J=$ $7.1 \mathrm{~Hz}, 3 \mathrm{H}) .{ }^{13} \mathrm{C}$ NMR (101 MHz, $\left.\mathrm{CDCl}_{3}\right) \delta 173.6(\mathrm{C}), 149.7$ (C), 148.7 (C), 128.5 $(\mathrm{CH}), 124.2(\mathrm{CH}), 66.7(\mathrm{C}), 62.9(\mathrm{CH}), 60.4\left(\mathrm{CH}_{2}\right), 40.7\left(\mathrm{CH}_{2}\right), 35.4\left(\mathrm{CH}_{2}\right), 34.2\left(\mathrm{CH}_{2}\right)$, $31.3\left(\mathrm{CH}_{3}\right), 27.3\left(\mathrm{CH}_{2}\right), 27.0\left(\mathrm{CH}_{3}\right), 26.2\left(\mathrm{CH}_{2}\right), 24.8\left(\mathrm{CH}_{2}\right), 14.4\left(\mathrm{CH}_{3}\right) . \mathrm{GC} \mathrm{t}_{\mathrm{R}}=26.48$ min. LRMS (EI) m/z (\%) 367 (10), 285 (5), 284 (15), 283 (100), 253 (3), 226 (33), 97 (5), 82 (9), 81 (42). HRMS (ES ${ }^{+}$) calcd for $\mathrm{C}_{19} \mathrm{H}_{29} \mathrm{~N}_{2} \mathrm{O}_{6} \mathrm{~S} 413.1746$, found 413.1740 .

\section{Acknowledgements}

We thank the Spanish Ministerio de Ciencia e Innovación for their financial support (CTQ2011-24165). I. B. acknowledges the Generalitat Valenciana for a predoctoral fellowship (ACIF/2011/159).

\section{Notes and references}

1 M. Yus, J. C. Gonzalez-Gomez and F. Foubelo, Chem. Rev., 2011, 111, 7774.

2 M. Yus, J. C. Gonzalez-Gomez and F. Foubelo, Chem. Rev., 2013, 113, 5595.

3 A. Yanagisawa, K. Ogasawara, K. Yasue and H. Yamamoto, Chem. Commun., 1996, 367.

4 I. Shibata, S. Miyamoto, S. Tsunoi, K. Sakamoto and A. Baba, Eur. J. Org. Chem., 2009, 3508.

5 L.-M. Zhao, S.-Q. Zhang, H.-S. Jin, L.-J. Wang and F. Dou, Org. Lett., 2012, 14, 886.

6 M.-Z. Chen, M. Mclaughlin, M. Takahashi, M.-A. Tarselli, D. Yang, S. Umemura and G.-C. Micalizio, J. Org. Chem., 2010, 75, 8048.

7 D.-C. Schmitt, J. Lee, A.-M. R. Dechert-Schmitt, E. Yamaguchi and M.-J. Krische, Chem. Commun., 2013, 49, 6096.

8 (a) I. Coldman and D. Leonori, J. Org. Chem., 2010, 75, 4069. (b) I. Coldman, S. P. Robinson and C. A. Baxter, Synlett, 2012, 23, 2405.

9 (a) B. Schlummer and J. F. Hartwig, Org. Lett., 2002, 4, 1471. (b) C. M. Haskins and D. W. Knight, Chem. Commun., 2002, 2724. (c) J. Elaridi, W. R. Jackson and A. J. Robinson, Tetrahedron: Asymmetry, 2005, 16, 2025. (d) C. M. Griffiths-Jones and D. 
W. Knight, Tetrahedron, 2010, 66, 4150. (e) B. J. vanLierop, W. R. Jackson and A. J. Robinson, Tetrahedron, 2010, 66, 5357.

10 M. Amjad and D. W. Knight, Tetrahedron Lett., 2006, 47, 2825.

11 (a) J. Gebauer and S. Blechert, Synlett, 2005, 2826. (b) F. Frebault, N. S. Simpkins and A. Fenwick, J. Am. Chem. Soc., 2009, 131, 4214. (c) H. Susuki and S. Aoyagi, Chem. Commun., 2011, 47, 7878.

12 N. Simpkins, I. Pavlakos and L. Male, Chem. Commun., 2012, 48, 1958.

13 M. Sugiura, C. Mori and S. Kobayashi, J. Am. Chem. Soc., 2006, 128, 11038.

14 (a) M. Rueping and A. P. Antonchick, Angew. Chem. Int. Ed., 2008, 47, 10090. (b)

W. D. Wulff and H. Ren, J. Am. Chem. Soc., 2011, 133, 5656.

15 For a precedent of 2-azonia-Cope rearrangement from a chiral template formed from a homoprenyl aminoalcohol and glyoxal, see: C. Agami, F. Couty and M. Poursoulis, Synlett, 1992, 847.

16 (a) J. C. Gonzalez-Gomez, M. Medjahdi, F. Foubelo and M. Yus, J. Org. Chem., 2010, 75, 6308. (b) J. C. Gonzalez-Gomez, F. Foubelo and M. Yus, Org. Synth., $2012,89,88$.

17 For amine 3a, the er was estimated by ${ }^{1} \mathrm{H}-\mathrm{NMR}$ analysis of compound $\mathbf{2 a}$, which was conveniently epimerized at the sulfur center. In the case of amine $\mathbf{3 b}$, the er was determined by HPLC analysis of the benzoyl amide. See the ESI for more details.

18 R. García, J. M. Seco, S. A. Vázquez, E. Quiñoá and R. Riguera, J. Org. Chem., 2006, 71, 1119. Details of the absolute configuration of selected examples are given in the ESI.

19 (a) M. Wakayama and J. A. Ellman, J. Org. Chem., 2009, 74, 2646; (b) V. K. Aggarwal, N. Barbero, E. M. McGarrigle, G. Mickle, R. Navas, J. R. Suárez, M. G. Unthank and M. Yar, Tetrahedron Lett., 2009, 50, 3482.

20 Both enantiomers are commercially available from various companies or can be prepared in large scale according to a reported procedure: D. J. Weix and J. A. Ellman, Org. Synth., 2005, 82, 157.

21 Compound 2a was previously described by us in: J. C. Gonzalez-Gomez, M. Medjahdi, F. Foubelo, M. Yus, J. Org. Chem. 2010, 75, 6308.

22 Prepared as described in: M. Altendorfer, A. Raja, F. Sasse, H. Irschik, D. Menche, Org. Biomol. Chem. 2013, 11, 2116.

23 Prepared from Ethyl hept-6-enoate by oxidative cleavage of the double bond with $\mathrm{OsO}_{4}$ cat. $/ \mathrm{NaIO}_{4}$. 
24 Prepared as described in: K. C. Nicolaou, V. A. Adsool, C. R. H. Hale, Org. Lett. 2010, 12, 1552. 